\title{
The Geometry of Super Riemann Surfaces
}

\author{
Steven B. Giddings ${ }^{1}$ and Philip Nelson ${ }^{2}$ \\ 1 Lyman Laboratory of Physics, Harvard University, Cambridge, MA 02138, USA \\ 2 Department of Physics, Boston University, Boston, MA 02215, USA
}

\begin{abstract}
We define super Riemann surfaces as smooth 2|2-dimensional supermanifolds equipped with a reduction of their structure group to the group of invertible upper triangular $2 \times 2$ complex matrices. The integrability conditions for such a reduction turn out to be (most of) the torsion constraints of $2 \mathrm{~d}$ supergravity. We show that they are both necessary and sufficient for a frame to admit local superconformal coordinates. The other torsion constraints are merely conditions to fix some of the gauge freedom in this description, or to specify a particular connection on such a manifold, analogous to the Levi-Civita connection in Riemannian geometry. Unlike ordinary Riemann surfaces, a super Riemann surface cannot be regarded as having only one complex dimension. Nevertheless, in certain important aspects super Riemann surfaces behave as nicely as if they had only one dimension. In particular they posses an analog $\hat{\partial}$ of the Cauchy-Riemann operator on ordinary Riemann surfaces, a differential operator taking values in the bundle of half-volume forms. This operator furnishes a short resolution of the structure sheaf, making possible a Quillen theory of determinant line bundles. Finally we show that the moduli space of super Riemann surfaces is embedded in the larger space of complex curves of dimension $1 \mid 1$.
\end{abstract}

\section{Introduction}

To describe a string moving through spacetime we introduce a two-dimensional parameter space $X$ and consider functions from $X$ to spacetime. Since the local dynamics of the string should depend only on the image of $X$ in spacetime, we require that the action functional describing the string be independent of the parametrization chosen. Locally it should also not depend on any extra information describing the auxiliary space $X$, for instance a metric or connection on $X$.

When we move beyond the naive picture of strings above to the more sophisticated picture of an arbitrary conformal field theory, $X$ takes on a more central role. Nevertheless the point made above are still valid: the classical action (when it exists) must be an intrinsically-defined functional of fields on $X$, defined without any use of the local shape of $X$. On the other hand, as is well known a smooth 2-manifold does not have enough information to permit us to define such 
an action, or even to define certain of the fields (the chiral ones) at all. What is needed is a middle ground between the "bare" surface $X$ and $X$ equipped with, say, a metric. This middle ground consists of taking $X$ to be a Riemann surface.

We can think about Riemann surfaces in two main ways. A smooth $X$ is a topological space with an atlas of patches and maps $\phi_{\alpha}: U_{\alpha} \rightarrow \mathbf{R}^{2}$ such that $g_{\alpha \beta}=\phi_{\alpha} \circ \phi_{\beta}^{-1}$ is smooth on $\phi_{\beta}\left(U_{\alpha} \cap U_{\beta}\right)$. If $\phi_{\alpha}$ have been taken so that $g_{\alpha \beta}: \mathbf{C} \rightarrow \mathbf{C}$ are all analytic, then they define a complex 1-manifold, or Riemann surface. In this case it makes sense to define a subset of all the smooth functions $\mathscr{A}$ on $X: \mathcal{O} \subseteq \mathscr{A}$ consists of functions which under each $\phi_{\alpha}$ become analytic functions on C. A collection of $\phi_{\alpha}$ is deemed equivalent to $\phi_{\alpha}^{\prime}$ if $\phi_{\alpha}=f_{\alpha} \circ \phi_{\alpha}^{\prime}$ for a set of analytic maps $f_{\alpha}: \mathbf{C} \rightarrow \mathbf{C}$.

In the second approach one defines a Riemann surface from a bare smooth 2 -surface by endowing the latter with additional geometrical structure. For example we can single out a subspace $\mathcal{O}$ of smooth complex functions and declare them to be the analytic ones. Since a compact $X$ has only one global analytic function (the constant), it is certainly not enough to give the space $\mathcal{O}(X)$ of these. Rather one must specify $\mathcal{O}$ as a "sheaf" on the open sets of $X$. Roughly speaking a sheaf is an assignment of a space of local functions to every open set $U$ of $X$, or more generally an assignment of a ring $\mathcal{O}(U)$ to each $U$. A ring has operations which behave like addition, subtraction, and multiplication, but the latter need not be invertible or even commutative. The various $\mathcal{O}\left(U_{\alpha}\right)$ must satisfy a few axioms which say that they fit together across intersections of open sets the same way the ordinary smooth functions do. ${ }^{1}$ In the present case $\mathcal{O}(U)$ must be isomorphic to the analytic functions on an open set of $\mathbf{C}$; in particular, it is commutative.

A more convenient construction than simply giving $\mathcal{O}$ explicitly is to split the complex tangent space $T_{c} X=(T X) \otimes \mathbf{C}$ into two complex-conjugate subspaces $T^{1,0} X \oplus T^{0,1} X$. This can be accomplished for example by giving a real tensor $J$, where $J(x): T_{x} X \rightarrow T_{x} X$ and $J^{2}=-1$; then $T^{1,0} X$ is the eigenspace of $J$ with eigenvalue $+i$. If we now think of tangent vectors as differential operators, we can define $(1)$ as being the smooth functions everywhere annihilated by any vector spanning $T^{0,1} X . \mathcal{O}(U)$ then has many local functions, but all of them can be generated by just one, a local complex coordinate $u$ on $U$.

If we like we can supply $X$ with more information than just $J$. For instance we can give a metric $\gamma$ on $X$, or even a frame $\left\{e_{1}, e_{2}\right\}$ for $T X$. Of course $e_{a}$ cannot be nondegenerate and smooth throughout $X$, but we can arrange for a choice on each patch $U_{\alpha}$ differing only by $S O(2)$ rotations on patch overlaps. Given such a frame we can introduce the complex vector field $e_{z}=\frac{1}{2}\left(e_{1}-i e_{2}\right)$ and its conjugate. Then $T^{1,0} X$ can be taken simply to be the space spanned by $e_{z}$, or equivalently we can let

$$
J=i e_{z} \otimes e^{z}-i e_{\bar{z}} \otimes e^{\bar{z}},
$$

where $\left\{e^{a}\right\}$ is a dual basis to $\left\{e_{a}\right\} . J$ is clearly real and globally defined, since it is unaffected by a local $S O(2)$ rotation of $\left\{e_{a}\right\}$. Indeed it is also unaffected by a local rescaling of $\left\{e_{a}\right\}$ and so depends only on the conformal class of the metric

${ }^{1}$ Sheaves are discussed in e.g. [1,2] 
$\gamma=\sum_{a} e^{a} \otimes e^{a}$, as desired. Since every metric can locally be coordinate-transformed to a form conformally related to the standard one, every Riemann surface is indeed locally indistinguishable from a patch of $\mathbf{C}$, as required and as was clear from the first approach.

Each of these two descriptions of Riemann surfaces has advantages. In the first we can perform all of our manipulations using only analytic functions: the $g_{\alpha \beta}$ are all analytic, as are the transition functions of any holomorphic bundles we may define and so on. Also we can if we like let the $g_{\alpha \beta}$ depend holomorphically on one or more complex parameters to define a holomorphic family of Riemann surfaces. On the other hand, we have had to cut $X$ into patches, obscuring the global meaning of the construction. Moreover, in a parametrized family the coordinate regions $\phi_{\alpha}\left(U_{\alpha}\right) \subset \mathbf{C}$ will themselves move around under a finite change of parameters. Also it is not clear a priori that a holomorphic family is the most general deformation possible. For all these reasons it is useful, though not essential, to have the second approach, which in fact is the older one. Here any construction is intrinsic if it is coordinate-invariant and depends only on the global given tensor field $J$. Equivalently we can choose a frame $e_{a}$ to represent $J$ by (1.1), with the understanding that any $e_{z}^{\prime}=f \cdot e_{z}$ must give the same answers. Here $f$ is a nowhere-zero complex function combining Weyl and $S O(2)$ transformations. In this approach one need not show that the space of $J$ tensors modulo diffeomorphisms represents the most general deformation - that's a definition. Instead one must show that the corresponding moduli space is in fact complex, since this is now not guaranteed.

In other words, to make $X$ a Riemann surface we equip it with a family of frames related by local transformations in $\mathbf{C}^{\times} \equiv G L(1, \mathbf{C})$. We say that we have reduced the structure group of $X$ from $G L(2, \mathbf{R})$ to $\mathbf{C}^{\times}$. Similarly in higher dimensions a complex manifold has a subspace $T^{1,0} X$ of $p$ complex dimensions, reducing the frame group from $G L(2 p, \mathbf{R})$ to the subgroup $G L(p, \mathbf{C})$ fixing $T^{1,0} X$. Here we find an important new subtlety, however. In one complex dimension any such reduction is locally equivalent to any other, in a sense to be made precise later. In several dimensions however some reductions are not equivalent to the standard one. The conditions for this "local flatness" to hold constrain the possible frames $\left\{e_{a}\right\}$ we can use to define a reduction.

In this paper we would like to see what happens to the preceding discussion when we pass to super Riemann surfaces (SRS). We have perhaps belabored the above well-known points because as we will see their generalization to SRS is not entirely straightforward. Again we seek a structure on a smooth surface of dimension $2 \mid 2$ which permits the definition of an invariant string action but which is locally trivial, i.e. locally equivalent to the standard surface. The analysis of SRS and their moduli along the first lines (as patching data) has been carried out in [3]-[7] and elsewhere. Here we will complement that work with a discussion following the second line-a differential-geometric treatment. This turns out to be an interpretation and extension of work begun in [8-13]. Some of these results were announced in [14]. A number of points made here and in [14] were independently made in the work of Baranov, Frolov, and Schwarz [15]. Others are already to be found in the original papers. 
In the next section we review some parts of supermanifold theory and the theory of reductions of structure groups. We then apply this to SRS, showing that the torsion constraints of $[8,9]$ are necessary conditions for a frame to be integrable into a superconformal structure. Next we establish the converse. Then in Sect. 5 we describe the fundamental complex on a SRS. This complex lets us write down the string action and defines the relevant DET bundles where partition functions live. Finally we study supermoduli space, its relation to the space of complex 1|1-dimensional curves, and its complex structure.

The present approach to SRS also lets us find good local holomorphic coordinates for supermoduli space. Details can be found in [16].

\section{Supermanifolds and Structure Groups}

A. Supermanifolds. We will first review two subjects: the theory of supermanifolds [17] and that of reductions of the structure group of an ordinary manifold [18]. Supermanifolds in a rudimentary form were first introduced to extend classical phase space to include spin degrees of freedom [19]. Later they were adopted as extensions of spacetime itself. The idea is to find a setting where supersymmetry transformations have the same geometrical status as ordinary spacetime translations. That is, flat superspace should have vector fields $P$ satisfying $[P, P]=0$ and generating symmetries, but also $Q$ satisfying the basic supersymmetry algebra $[Q, P]=0,[Q, Q] \sim P$. For this to work with $Q_{\mu}=$ $\left(\partial / \partial \psi^{\mu}\right)+\cdots$ we must ascribe to the coordinate $\psi^{\mu}$ a spinorial character.

An ordinary smooth manifold $X$ of dimension $p$ can be thought of as a topological space and an assignment to every open set $U$ of a $\operatorname{ring} \mathscr{A}(U)$, the smooth functions on $U$. On small enough $U, \mathscr{A}(U)$ is generated by $p$ independent functions - real coordinates $y^{m}$ of $X$. To obtain a supermanifold $\hat{X}$ we start with the same topological space but assign to it a sheaf of rings $\hat{\mathscr{A}}$ larger than $\mathscr{A}$. $\hat{\mathscr{A}}$ is $\mathbf{Z}_{2}$-graded; it is not commutative but rather graded-commutative. In fact we will require that over small enough open sets $U \subseteq X$ we have

$$
\hat{\mathscr{A}}(U) \cong \mathscr{A}(U) \otimes \wedge(V),
$$

where $V$ is a $q$-dimensional vector space and $\wedge(V)$ its exterior algebra. $\mathscr{A}$ consists of the ordinary functions on an ordinary smooth manifold $X$. Thus if $\psi^{\mu}$ are a basis of $V$, then $\hat{\mathscr{A}}(U)$ is generated by the images under (2.1) of $y^{m}$ and $\psi^{\mu}$. We agree to treat these two kinds of generators on an equal basis, and we call both of them "coordinates." The isomorphism (2.1) is not natural, so that across patch boundaries we will in general get transition functions. These functions must preserve the usual multiplication on $\mathscr{A}$ and $\wedge(V)$, as well as the parity of the generators $y^{m}$ and $\psi^{\mu}$.

A general element of $\hat{\mathscr{A}}$ is now locally of the form

$$
f_{1}\left(y^{m}\right)+f_{2 \mu}\left(y^{m}\right) \psi^{\mu}+f_{3 \mu \nu}\left(y^{m}\right) \psi^{\mu} \psi^{v}+\cdots,
$$

which we will denote by $f\left(y^{m}, \psi^{\mu}\right)$ in keeping with our resolve to think of all the elements of $\mathscr{A}$ as generalized "functions" on some "space." Also we will sometimes refer to $y^{m}, \psi^{\mu}$ collectively as $y^{M}$, where the generic index $M$ runs over $p+q$ values. 
The idea of characterizing a space by its ring of functions is very natural mathematically. It is also physically natural, for example in Hamiltonian dynamics where primary emphasis is placed on the ring of observables (in this case actually an algebra) and phase space itself plays a secondary role. In the latter case one is inevitably led to extend the ring in order to describe fermions; one then notes that most of the formulas of ordinary geometry continue to make sense in this setting [20].

While the isomorphism (2.1) is not natural, still we can canonically construct the ordinary manifold $X$ from $\hat{X}$. Note that the functions in (2.2) with $f_{1}=0$ are the same in any coordinate system; they can be invariantly characterized as the nilpotents of $\mathscr{A}(U)$. The subsheaf $N \subset \mathscr{A}$ of nilpotents is in fact an ideal, so we can recover $\mathscr{A}$ as the quotient

$$
\mathscr{A} \cong \hat{\mathscr{A}} / N
$$

$\mathscr{A}$ defines an ordinary smooth manifold $X$, sometimes called the body of $\hat{X}$. Similarly given a supermanifold $\hat{X}$ we can always build a bundle $E_{\hat{X}}$ over its body $X$. We simply take the local sections of $E_{\hat{X}}$ to be

$$
\mathscr{E}_{\hat{X}} \equiv N / N^{2}
$$

In any coordinate system these are roughly "the functions with only one $\psi . " E_{\hat{X}}$ is indeed a bundle of dimension $q$, by (2.1). We can also reverse this prescription to construct a large class of supermanifolds: if $E$ is a vector bundle on an ordinary manifold $X$ then locally $\left.E\right|_{U} \cong \mathbf{R}^{q}$. Hence taking $\hat{\mathscr{A}}$ to be the sections of $\wedge E$ we see that (2.1) is satisfied. We will also write $\hat{\mathscr{A}}=\wedge \mathscr{E}$, where $\mathscr{E}$ is the sheaf of sections of $E$. If $\hat{\mathscr{A}}$ can be taken to be of this form we say that $\hat{X}$ is split. (The terminology is explained in [21].) Even if $\hat{X}$ is split there may be several different splittings. $^{2}$

A more practical criterion of splitness is the following. If we have chosen trivializations of $\hat{\mathscr{A}}$ on $U_{1}, U_{2}$, i.e. isomorphisms $\phi_{\alpha}: \hat{\mathscr{A}}\left(U_{\alpha}\right) \rightarrow \mathscr{A}\left(U_{\alpha}\right) \otimes \wedge\left(\mathbf{R}^{q}\right)$, then we get a ring isomorphism of $\left.\mathscr{A}\right|_{U_{1} \cap U_{2}} \otimes \wedge\left(\mathbf{R}^{q}\right)$ with itself. These transition functions always respect the overall parity of each coordinate, since by definition $\hat{\mathscr{A}}$ has a globally defined $\mathbf{Z}_{2}$ grading. If we can arrange that the transition functions are always of the form

$$
y^{\prime m}=f^{m}\left(y^{n}\right), \quad \psi^{\prime \mu}=g_{v}^{\mu}\left(y^{m}\right) \psi^{v},
$$

then $\hat{X}$ is split, and conversely. If on the other hand, $y^{\prime m}, \psi^{\prime \mu}$ necessarily have the form

$$
\begin{aligned}
& y^{\prime m}=f^{m}\left(y^{n}\right)+k_{\mu \nu}^{m}\left(y^{n}\right) \psi^{\mu} \psi^{v}+\cdots, \\
& \psi^{\prime \mu}=g_{v}^{\mu}\left(y^{m}\right) \psi^{v}+h_{v \kappa \lambda}^{\mu}\left(y^{m}\right) \psi^{v} \psi^{\kappa} \psi^{\lambda}+\cdots,
\end{aligned}
$$

then $\hat{X}$ does not split. If we consider not a single manifold $\hat{X}$ but a family parametrized by some superspace $\hat{Z}$, then we can have more generally

$$
\psi^{\prime \mu}=\Gamma^{\mu}\left(y^{m}\right)+g_{v}^{\mu}\left(y^{m}\right) \psi^{v}+\cdots,
$$

\footnotetext{
${ }^{2}$ An analogy is to spin structures: even if a space admits a spin structure, there may be several inequivalent choices
} 
where $\Gamma^{\mu}$ is an odd function of $y^{m}$ and of the coordinates of $\hat{Z}$ and $g_{v}^{\mu}$ is an even function. It is now clear that the above construction of a bundle $E_{\hat{X}}$ from an arbitrary supermanifold $\hat{X}$ discards some of the information in (2.5), namely the pieces $k_{\mu \nu}^{m}, h_{v \kappa \lambda}^{\mu}, \ldots$ of the transition function. Thus if we begin with an arbitrary $\hat{X}$, build $E_{\hat{X}}$, and then build $\hat{X}^{\prime} \equiv\left(X, \wedge \mathscr{E}_{\hat{X}}\right)$, we find that while $\hat{X}^{\prime}$ is always split, it is not necessarily isomorphic to the original $\hat{X}^{3}$

A simple split supermanifold which we will use later is obtained when $X$ is a real 2-surface and $E$ is a spinor bundle. Then $\psi^{\mu}, \mu=1,2$ are taken to be two real local spinor sections, everywhere linearly independent. Clearly $y^{m}, \psi^{\mu}$ generate all of $\hat{\mathscr{A}}$ and satisfy (2.4).

A fundamental theorem asserts that when we consider smooth supermanifolds we find that every one is split, though not necessarily in a natural way [22,23]. In the complex-analytic case, however, this need no longer be so. If the family has odd parameters then $\Gamma$ in (2.6) can be unremovable. Even with only even parameters an obstruction to splitting can arise [21]. The situation is reminiscent of the theory of complex line bundles on a Riemann surface: any two bundles of the same degree are indistinguishable as smooth bundles, but it may not be possible to identify them using a holomorphic isomorphism. Indeed the classes of holomorphic line bundles of degree zero form a Lie group, the Picard group $\mathrm{Pic}_{0}$.

A map between two supermanifolds $\hat{f}: \hat{X} \rightarrow \hat{Y}$ is a homomorphism from $\hat{\mathscr{A}}_{\hat{Y}}$ back to $\hat{\mathscr{A}}_{\hat{X}}$. More precisely $\hat{f}$ consists of an ordinary continuous map $f: X \rightarrow Y$, and for each open $U \subseteq Y$ a homomorphism $f^{*}: \hat{\mathscr{A}}_{\hat{Y}}(U) \rightarrow \hat{\mathscr{A}}_{\hat{X}}\left(f^{-1} U\right)$ respecting the $\mathbf{Z}_{2}$-grading. One important map is the inclusion

$$
\hat{\imath}: X \hookrightarrow \hat{X}
$$

where we regard $X$ itself as a trivial supermanifold. Here the map on the body, $l: X \rightarrow X$ is the identity, while $\imath^{*}: \hat{\mathscr{A}} \rightarrow \mathscr{A}$ is the natural map defined by (2.3). It is important to note, however that in general no natural projection $\hat{\pi}: \hat{X} \rightarrow X$ exists. If we are given a splitting of $\hat{X}$, however, then a simple choice for $\hat{\pi}$ exists: take $\pi^{*}$ to include $\mathscr{A}$ into $\hat{\mathscr{A}}$ as the zeroth wedge power of the bundle $E$ defining $\hat{X}$.

As in ordinary geometry we can define vector fields directly in terms of the ring of functions [24]: a vector field is a derivation of $\hat{\mathscr{A}}$, with now the appropriate grading behavior, i.e.

$$
v(f g)=(v f) \cdot g+(-)^{|v| \cdot|f|} f \cdot(v g)
$$

where $v f$ means the partial derivative of $f$ along the vector field $v$. We will use only vectors satisfying (2.8), i.e. vectors which act from the left. Vectors like $\partial / \partial y^{m}$ and $\psi^{\nu}\left(\partial / \partial \psi^{\mu}\right)$ are called even sections of the tangent sheaf $\hat{\mathscr{T}}$ of $\hat{X} ; \partial / \partial \psi^{\mu}$ and $\psi^{\mu}\left(\partial / \partial y^{m}\right)$ are odd sections of $\mathscr{T}$. Here $\psi^{v}\left(\partial / \partial \psi^{\mu}\right)$ is the derivation of $\hat{\mathscr{A}}$ obtained by acting on $f\left(y^{m}, \psi^{\mu}\right)$ from the left with $\partial / \partial \psi^{\mu}$, then multiplying on the left by $\psi^{v}$. More generally we can multiply any vector field by any function in $\hat{\mathscr{A}}$. We therefore say that $\hat{\mathscr{T}}$ is a (graded left) " $\hat{\mathscr{A}}$-module of rank $p \mid q$." One can also regard $\hat{\mathscr{T}}$ as the sheaf of sections of a bundle $T \hat{X}$. If $\hat{X}$ is split, then $T \hat{X}$ decomposes

\footnotetext{
${ }^{3}$ Even if $\tilde{X}$ was split to begin with, $\hat{X}^{\prime}$ will not be canonically isomorphic to $\hat{X}$
} 
into two ordinary bundles $T X \oplus E^{*}$ over $X$, where $E^{*}$ denotes the dual to $E .^{4}$

One can generalize $\hat{\mathscr{T}}$ to an arbitrary sheaf of graded $\hat{\mathscr{A}}$ modules $\hat{\mathscr{F}}$. This means that the super functions (2.2) can multiply the sections of $\hat{\mathscr{F}}$ in the usual way. If moreover $\hat{\mathscr{F}}$ is locally free, we say that it defines a vector bundle over $\hat{X}$. The latter condition just means that locally the sections of $\hat{\mathscr{F}}$ look like $n$-tuples of functions in $\hat{\mathscr{A}}$, with the usual vector space scalar multiplication, and that the transition functions across patches preserve the grading, so that $\hat{\mathscr{F}}$ has a well-defined dimension $r \mid s$, where $r+s=n$. In particular $\hat{\mathscr{T}}$ is a vector bundle of rank $p \mid q$.

$\hat{\mathscr{T}}$ has a richer structure than an arbitrary bundle $\hat{\mathscr{F}}$; in the usual way one can turn it into a sheaf of algebras by defining the graded Lie bracket: $\left[v, v^{\prime}\right] f=v\left(v^{\prime} f\right)-$ $(-)^{|v| \cdot\left|v^{\prime}\right|} v^{\prime}(v f)$.

By the usual rules of linear algebra on $\mathbf{Z}_{2}$-graded spaces one can define the dual space $T^{*} \hat{X}$ of forms. We will use the convention for dual bases that $\left\langle E_{A}, E^{B}\right\rangle=\delta_{A}^{B}$; in particular,

$$
\left\langle\frac{\partial}{\partial y^{M}}, d y^{N}\right\rangle=\delta_{M}^{N},
$$

where $y^{M}=\left(y^{m}, \psi^{\mu}\right)$. Thus if $E_{A}=E_{A}{ }^{N}\left(\partial / \partial y^{N}\right)$ and $E^{A}=d y^{N} E_{N}{ }^{A}$, we get $E_{A}{ }^{N} E_{N}{ }^{B}=\delta_{A}^{B}$. We will always adhere to this $\searrow$ rule for index summation, in anticipation of applications to spinors.

One can build a de Rham complex as follows [9]. $\Omega$ is generated by the symbols $d y^{m}, d \psi^{\mu}$ over $\hat{\mathscr{A}}$. It is bigraded. Functions $f \in \hat{\mathscr{A}}$ have grading $(p, \sigma)=$ $(0,|f|)$, where $|f|$ is the parity of $f$. The symbol $d$ increases the grading $p$ and does not affect $\sigma . \Omega^{\prime}$ is subject to the relations

$$
\omega \wedge \omega^{\prime}=(-)^{p p^{\prime}}(-)^{\sigma \sigma^{\prime}} \omega^{\prime} \wedge \omega .
$$

Thus $d y^{1} d y^{2}=-d y^{2} d y^{1}, \quad d \psi^{1} d \psi^{2}=+d \psi^{2} d \psi^{1}$, but $d y^{1} d \psi^{1}=-d \psi^{1} d y^{1}$, in contrast to the definition in [17]. Define $d: \Omega^{p} \rightarrow \Omega^{p+1}$ by

$$
\begin{array}{r}
d f=d y^{N} \frac{\partial f}{\partial y^{N}} \equiv d y^{N} \cdot \partial_{N} f, \\
d\left(d y^{M} \omega_{M}\right)=d y^{M} \wedge d y^{N}\left(\partial_{N} \omega_{M}\right),
\end{array}
$$

for a function $f$ and 1-form $\omega$. Thus $d$ acts from the right. It dies not agree with the usual $d$ when $\hat{X}$ has no odd coordinates. This unfortunate situation is forced on us by our insistence on the above $\searrow$ summation rule, which says e.g. that one-forms must be written as $\omega=d x^{N} \omega_{N}$. With these rules $d^{2}=0$. For a two-form as usual we define

$$
\omega \equiv \frac{1}{2} d x^{M} d x^{N} \omega_{N M} \equiv \frac{1}{2} E^{B} E^{A} \omega_{A B},
$$

where $E^{A}$ is any frame for $T^{*} \hat{X}$. Then $(d \omega)_{N M}=2 \partial_{[N} \omega_{M]} \equiv \partial_{N} \omega_{M}-(-)^{N M} \partial_{M} \omega_{N}$.

All of the definitions so far have complex analogs. First, the sheaf of real rings $\hat{\mathscr{A}}$ can as usual be complexified to $\hat{\mathscr{A}}_{c}=\hat{\mathscr{A}} \otimes \mathbf{C}$, the complex functions on a real

${ }^{4}$ In terms of sheaves, we say that $\hat{\mathscr{T}} \cong \mathscr{T} \oplus \mathscr{E}^{*}$ as sheaves of $\mathscr{A}$-modules 
supermanifold. $\hat{A}_{c}$ has a natural involution, in which $\overline{f(y, \psi) \otimes w}=f(y, \psi) \otimes \bar{w}$ for $f \in \hat{\mathscr{A}}$. Then

$$
\overline{g h}=\bar{g} \bar{h}
$$

for $g, h \in \hat{\mathscr{A}}_{c}$, so $\hat{\mathscr{A}} \subseteq \hat{\mathscr{A}}_{c}$ is the subring of self-conjugate functions.

A remark about (2.9) is in order. One sometimes encounters the distinct convention $\overline{g h}=\bar{h} \bar{g}$. The latter is appropriate for functional superspaces over which one performs Minkowski space path integrals. It guarantees that the functional integral for $\left\langle\psi(\sigma, t) \psi^{\dagger}\left(\sigma^{\prime}, t^{\prime}\right)\right\rangle$ gives the complex conjugate of the one for $\left\langle\psi\left(\sigma^{\prime}, t^{\prime}\right) \psi^{\dagger}(\sigma, t)\right\rangle$. In euclidean space $\psi$ and $\bar{\psi}$ are independent, so this reasoning does not apply and we are free to impose (2.9). With (2.9) the conjugate of a vector again acts from the left; it is defined by $\bar{v} f \equiv \bar{v} \bar{f}$.

A complex supermanifold $\hat{X}$ is a supermanifold with a global notion of which functions are holomorphic. Specifically $\hat{X}$ is a topological space $X$ ringed by a sheaf $\hat{\mathcal{O}}$ such that

- each $\hat{\mathcal{O}}(U)$ is a complex graded ring,

- $(X, \mathcal{O})$ is an ordinary complex manifold, where $\mathcal{O}=\hat{\mathcal{O}} / N$, and

- locally we have

$$
\hat{\mathcal{O}} \cong \mathcal{O} \otimes \wedge(W)
$$

for some complex vector space $W$.

Equation (2.10) is an isomorphism of complex vector spaces. In particular, on two overlapping patches (2.10) implies transition functions in which the odd complex coordinates $\theta^{\mu}$ are expressed as power series in $\theta^{\prime \mu}$, with no $\bar{\theta}^{\prime \mu}$ terms. We call such functions holomorphic in $\theta$.

Thus the functions on $\hat{X}$ are generated by $p$ commuting generators $u^{m}$ and $q$ anticommuting $\theta^{\mu} . \hat{X}$ is split if $\hat{\mathcal{O}} \cong \wedge \mathscr{E}$, where now $\mathscr{E}$ is the sheaf of holomorphic sections of a holomorphic bundle $E$. The derivations $\hat{\mathscr{T}}$ of $\hat{\mathcal{O}}$ are holomorphic vector fields. They form an $\hat{\mathcal{O}}$-module, since a vector field remains holomorphic when multiplied by a function in $\hat{\mathcal{O}}$.

Given a complex supermanifold we can find a corresponding smooth manifold of dimension $2 p \mid 2 q$ as follows. First we find the smooth ordinary manifold $(X, \mathscr{A})$ associated to $(X, \mathcal{O})$ in the usual way. Since the direct sum of any complex vector space with its conjugate is naturally the complexification of some real vector space,

$$
W \oplus \bar{W} \cong V \otimes_{\mathbf{R}} \mathbf{C},
$$

we can define a real bundle $\mathscr{E}_{r}$ of dimension $2 q$ from $\mathscr{E}_{\hat{X}} \oplus \overline{\mathscr{E}_{\hat{X}}}$. We can then define $\hat{\mathscr{A}}$ from $\mathscr{E}_{r}$ using the transition functions of the original $\hat{\mathcal{O}}$. Conversely we can consider the problem of taking a real $\hat{X}$ and finding an $\hat{\mathcal{O}} \subseteq \hat{\mathscr{A}}_{c}$, which makes $\hat{X}$ complex, analogously to the ordinary case discussed in the introduction. This we will do in the following sections.

B. Structure Groups. Given an ordinary real manifold $X$ it is useful to consider at each point $P \in X$ the set of all frames, that is all $n$-tuples of tangent vectors $\left\{E_{a}\right\}$ spanning $T_{P} X$. These frames do not themselves form a group, but the group $G L(n, \mathbf{R})$ acts to permute them. 
If $X$ is equipped with additional structure, then at each point certain of the frames may be singled out. Conversely, the specification of a subclass of "good" frames at each point of $X$ can induce some additional object on $X$. For example, in special relativity we define a class of good frames, those which come from inertial coordinate systems. Each such frame defines many geometrical objects, for example its "time" direction, but most of these are not common to all the good frames and so not intrinsically defined. One object is common to all inertial frames, however: one proves that the metric, originally defined using coordinates, is in fact invariant.

Thus the choice of a class of good frames permuted by $O(3,1)$ defines a metric, while conversely the choice of a metric defines the class of frames in which it takes its canonical form.

There are other classical examples of reductions from $G L(n, \mathbf{R})$ to a subgroup $G$. For example the choice of our almost-complex structure defines a class of good frames for the complex tangent space, where the first $n / 2$ vectors $E_{z^{\prime}}$ lie in $T_{P}^{1,0} X$, while the remaining $n / 2, E_{\bar{z}^{\prime}}$ are their complex conjugates. Two such frames differ by a matrix of the form

$$
\left(\begin{array}{ll}
A & 0 \\
0 & \bar{A}
\end{array}\right), \quad A \in G L\left(\frac{n}{2}, \mathbf{C}\right) .
$$

Conversely, given such a reduction we can define

$$
J=i\left(\sum_{i} E_{z^{2}} \otimes E^{z^{2}}-\text { c.c. }\right),
$$

which clearly does not change if we replace $E_{z^{i}}$ by $U_{i}^{j} E_{z^{\prime}}$. As usual $\left\{E^{z^{i}}\right\}$ is the basis of $T^{*(1,0)} X$ dual to $\left\{E_{z^{\prime}}\right\}$.

A third example we will need is the choice of a distribution. Suppose $X$ is equipped with a special subbundle $\mathscr{D}$ of its tangent space. Let $\mathscr{D}$ have dimension $k<n$. Then we can declare the good frames to be those for which the last $k$ vectors $E_{\alpha}, \alpha=1, \ldots, k \operatorname{span} \mathscr{D}$, while the remaining $E_{a}, a=1, \ldots, n-k$ complement $\mathscr{D}$. Now two such frames differ by a matrix of the form

$$
U=\left(\begin{array}{ll}
A & \Gamma \\
0 & B
\end{array}\right),
$$

where $A$ and $B$ are invertible. Conversely given such a reduction let $\mathscr{D}$ be the space spanned at each point by the last $k$ vectors of any frame. By construction $\mathscr{D}$ depends only on the reduction chosen, not on the representative frame. Unlike the previous two cases, however, in general $\mathscr{D}$ cannot be regarded as being defined by a tensor such as a metric or $J_{A}{ }^{B}$.

Suppose we have a manifold $X$ and a reduction to a group $G$. Another manifold $Y$ with reduction is called equivalent to $X$ if there is a diffeomorphism $\phi: X \rightarrow Y$ which sends every good frame of $T X$ to a good frame of $T Y$ [18].

In general the question of whether two reductions are equivalent is a very difficult one. A much easier question asks whether for each $P \in X$ one can find a neighborhood $U$ of $P$ and a diffeomorphism $\phi: U \rightarrow V$ such that the reduction on $U$ is equivalent to the one on $V$. In particular one can let $Y$ be $\mathbf{R}^{n}$, with some 
standard reduction. If $X$ is locally equivalent to $Y$, then we say that the given reduction on $X$ is flat. Alternately, flatness means that there exist local coordinates on $U$ in which the reduction takes a standard form.

In the first example above, $Y$ can be $\mathbf{R}^{n}$ with the usual flat metric. (This example motivates the general use of the term "flat.") The special coordinates near a point $P$ are then the inertial coordinates for the given metric near $P$; these exist if and only if the metric is flat in the usual sense. In the second example, $Y$ can be $\mathbf{R}^{n}$ with the standard identification with $\mathbf{C}^{n / 2}$. The special coordinates $\left\{u^{i}\right\}$ are then the holomorphic coordinates. Two different sets of holomorphic coordinates differ by a holomorphic diffeomorphism. In the third example $Y$ is $\mathbf{R}^{n}$ and $\mathscr{D}$ is everywhere spanned by the last $k$ coordinate differentials.

We can get a necessary condition for local flatness of a reduction as follows. Suppose we present a reduction, or $G$-structure, locally by some specific family of frames $E_{A}$ vaying smoothly near $P$. The full set of good frames is then obtained at each point as $U_{A}{ }^{B} E_{B}$ where $U \in G$. Consider the tensor defined by the Lie brackets

$$
\left[E_{A}, E_{B}\right]=t_{A B}{ }^{C} E_{C} .
$$

For each of the three examples we can arrange that the "structure functions" $\hat{t}_{A B}{ }^{C}$ on the standard space $Y$ are all identically zero, simply by taking $\hat{E}_{A}$ to be the differentials of a coordinate system. More generally suppose that the $\hat{t}_{A B}{ }^{C}$ of a standard $G$-structure are all constants. If the reduction on $X$ is locally equivalent to the standard one, then there will exist a local frame $E_{A}$ on $X$ pulled back from $Y$, and hence satisfying $t_{A B}{ }^{C}=$ const $=\hat{t}_{A B}{ }^{C}$.

An arbitrary frame $E_{A}^{\prime}=U_{A}{ }^{B} \hat{E}_{B}$, where $U: X \rightarrow G$, will in general have $t_{A B}^{\prime}{ }^{C}$ differing from $\hat{t}_{A B}{ }^{C}$. It can happen, however, that certain components of $t_{A B}^{\prime}{ }^{c}$ are unchanged after any such "gauge" transformation. Each such gauge-invariant component must take its standard value in order for $X$ to be locally flat. The smaller the group $G$, the larger will be the set of these $G$-invariant conditions.

In the examples, one first notes that if $G=O(n)$ then there are no invariant components of $\hat{t}_{A B}{ }^{c}$, and hence no conditions of the type we are considering. This just means that as far as first derivatives are concerned, every metric is the same as any other. It is of course this fact which makes Riemannian geometry suitable for implementing the Equivalence Principle of general relativity. If $G=G L(n / 2, \mathbf{C})$. however, we have $E_{z^{i}}^{\prime}=U_{i}^{j} E_{z^{j}}$ and (since $\hat{E}_{z^{J}}=\partial / \partial u^{j}$ )

$$
\left[E_{z^{i}}^{\prime}, E_{z^{j}}^{\prime}\right]=U_{i}{ }^{k}\left(\frac{\partial U_{j}^{l}}{\partial u^{k}}\right) \hat{E}_{z^{l}}-(i \leftrightarrow j)=U_{i}^{k}\left(\partial_{u^{k}} U_{j}^{l}\right)\left(U^{-1}\right)_{l}^{m} E_{z^{m}}^{\prime}-(i \leftrightarrow j) .
$$

Thus while $t_{z^{i} z^{\prime}}^{\prime z^{m}}$ is complicated, $t_{z^{2} z}^{\prime}{ }^{z^{k}}$ remains zero. Hence an almost-complex structure can be flat (locally identical to $\mathbf{C}^{n / 2}$ ) only if it satisfies

$$
t_{z^{i} z^{z^{2}}}^{z^{h}} \equiv 0
$$

a simple repackaging of the condition that its Nijenhuis tensor should vanish [25]. Similarly when $X$ has a distribution one shows that $\mathscr{D}$ can be flat (locally isomorphic to $T \mathbf{R}^{k} \subseteq T \mathbf{R}^{n}$ ) only if

$$
t_{\alpha \beta}{ }^{c}=0 ; \quad \alpha, \beta=1, \ldots, k ; \quad c=1, \ldots, n-k .
$$


This is the familiar Frobenius condition.

It is clear from the first example that our necessary condition does not in general suffice to conclude that a $G$-structure is flat. For $G=O(n)$ the condition is vacuous, and yet not every metric is flat! However, in both of the other examples an "integrability theorem" tells us that in fact there are no further obstructions: a reduction satisfying (2.15) (respectively (2.16)) defines a complex manifold (respectively a foliation of $X$ ).

If a $G$-structure is flat to first order then in some cases we can use it to define a connection, regardless of whether it is truly flat. Consider again the case of $O(n)$. If a given reduction admits an orthonormal frame of commuting vector fields near each point $P \in X$, then it is flat. Even if no such frame exists locally, there still may exist near each point $P$ a frame such that $t_{A B}{ }^{C}=0$ at $P$. We can then declare that the coefficients of the connection vanish in such a frame. In more invariant language, the condition $t(P)=0$ imposes conditions on the first derivatives of $E_{A}$ about $P$. If these conditions fix the derivatives completely then we get a map from tangent vectors on $X$ to tangents on its frame bundle. Such a "lift" determines a connection.

In the case of $O(n)$ we have uniquely defined a connection by the fact that it vanishes in a locally inertial frame, a result sometimes known as Levi-Civita's theorem. We will find an analogous result for super Riemann surfaces, but with only some, not all, of $t$ set to zero.

\section{Superconformal Structures}

We begin with the definition of super Riemann surfaces appearing in $[26,15,6]$ : A SRS is a 1|1-dimensional complex manifold $\hat{X}$ equipped with a holomorphic subbundle $\mathscr{D} \subseteq T \hat{X}$. More precisely $\mathscr{D}$ is a subsheaf of rank $0 \mid 1$ in $\mathscr{T}_{\hat{X}}$. Furthermore we demand that $\mathscr{D}$ satisfy a nondegeneracy condition: given any section $D$ of $\mathscr{D}$, the vector $[D, D]$ must be non-vanishing and everywhere linearly independent of $D$. This is as far as we can get from satisfying the Frobenius condition (2.16). Thus there is no sense in which $\mathscr{D}$ singles out a special function $\eta$ of the holomorphic coordinates $u$ and $\theta$ as a "good" coordinate, the way $T^{1,0} X$ distinguishes $u$ from $\bar{u}$ on an ordinary Riemann surface. Nevertheless we will see that in some ways a SRS behaves as if it had just one dimension.

In practice the notion of a single SRS is not very interesting. This is a general rule in super geometry: the points of a supermanifold are not the whole story. Indeed if we define "points" as maximal ideals of $\hat{\mathscr{A}}$, we see that they are simply the points of the underlying manifold $X$. Similarly when we define a class of super-objects we typically get a superspace describing them. If we look only at individual SRS, for example, we trivially find that they are all split: with only one odd dimension (2.5) always reduces to (2.4). (Recall that the transition functions involve $\theta$ but not $\bar{\theta}$.) Indeed it is easy to see that the set of all SRS is just the spinmoduli space, the space of all Riemann surfaces equipped with a spin structure [5]. Given such a surface we simply choose any local coordinate $u$ and take the generator $\theta$ to be $(d u)^{1 / 2}$ in the given spin structure. We can then let

$$
D \equiv \partial_{\theta}+\theta \partial_{u}
$$


span $\mathscr{D}$. Beginning with a differential $u^{\prime}$ gives $D^{\prime}=\left(d u / d u^{\prime}\right)^{-1 / 2} D$, and so $\mathscr{D}$, the space spanned by $D$, is well defined.

To see all of supermoduli space, including its odd coordinates, one must consider families of SRS parametrized by both even and odd coordinates. Such a family is essentially a complex manifold $\hat{W}$ of dimension $p+1 \mid q+1$ together with a projection to a $p \mid q$-dimensional parameter space $\hat{Z}$, whose fibers are SRS [6]. $\mathscr{D}$ is now a subbundle of the vertical (or "relative") tangent to $\hat{W}, \mathscr{T}_{\hat{W} \mid \hat{Z}}$. It satisfies the same consistency condition as before. We will sometimes use shorthand notation in which $\hat{Z}$ is suppressed, but it will always be in the background, the "source" of anticommuting parameters in formulas like (2.6).

Clearly a SRS is nothing but a reduction of the structure group of $\hat{X}$. Originally this group is $G L(2 \mid 2, \mathbf{R})$, the real $4 \times 4$ graded matrices of the block form

$$
U=\left(\begin{array}{ll}
U_{1} & r_{1} \\
r_{2} & U_{2}
\end{array}\right),
$$

where $U_{i}$ are $2 \times 2$ matrices of even functions and $Y_{i}$ are odd. A matrix in which every entry has its natural parity will be called "graded-even" [17]. One can take $U$ to be of this form because even a "bare" $\hat{X}$, with only its supermanifold structure, has a class of frames compatible with its grading. These are the frames $\left\{E_{a}, E_{\alpha}\right\}$ in which the first two vectors are even sections of $\mathscr{T}$ while the last $q$ are odd. Such frames are related by the graded-even transformations (3.1). A transformation of this form is invertible whenever the "reduced" matrices $U_{i, r d}$, obtained by setting all the Grassmann generators to zero, are invertible as ordinary matrices.

A frame can be used to reduce the structure group from $G L(2 \mid 2, \mathbf{R})$ to $G L(1 \mid 1, \mathbf{C})$. Exactly as in the classical case, we can make all the tangent spaces complex and consider base $E_{z}, E_{+}, E_{\bar{z}}, E_{-}$with $E_{\bar{z}}=\overline{E_{z}}, E_{-}=\overline{E_{+}}$. Two such bases are deemed equivalent if they are related by

$$
\left[\begin{array}{l}
E_{z}^{\prime} \\
E_{+}^{\prime} \\
E_{\bar{z}}^{\prime} \\
E_{-}^{\prime}
\end{array}\right]=\left(\begin{array}{ll}
U & 0 \\
0 & \bar{U}
\end{array}\right)\left[\begin{array}{l}
E_{z} \\
E_{+} \\
E_{\bar{z}} \\
E_{-}
\end{array}\right] .
$$

Note that we have rearranged and rows and columns of (3.1). $U$ is now a $2 \times 2$ graded-even matrix, invertible in the sense described above.

One can also introduce a standard space $\mathbf{C}^{1 \mid 1}$ with standard frame $\hat{E}_{z}=\partial / \partial u$, $\hat{E}_{+}=\partial / \partial \theta$. A given frame is then equivalent to the standard one up to first derivatives if and only if it satisfies [27]

$$
t_{z+}{ }^{\bar{z}}=t_{z+}{ }^{-}=t_{++}{ }^{\bar{z}}=t_{++}{ }^{-}=0,
$$

the super version of (2.15). These equations imply also the conjugate relations $t_{--}{ }^{z}=0$, etc. because of our choice of the convention (2.9). Note that (3.3) is more restrictive than the case of two ordinary complex dimensions, since $\left[E_{+}, E_{+}\right]$is not automatically zero.

Given a frame everywhere satisfying (3.3) we expect that an integrability theorem will imply that to all orders it is equivalent to $\mathbf{C}^{1 \mid 1}$. The appropriate modification of the classical theorem appears in the next section. 
So far we have considered a frame as defining an almost-complex structure on $\hat{X}$. Reducing the structure group still further gives an "almost-superconformal" structure, as follows. We consider two frames to be equivalent only when they are related by (3.2) with $U$ preserving the space spanned by $E_{+}$. That is, $U$ lies in the supergroup

$$
G_{1}=\left\{\left(\begin{array}{cc}
A & \Gamma \\
0 & B
\end{array}\right) ; \quad A, B \text { invertible }\right\} .
$$

A class of frames related by $G_{1}$ defines a subspace $\mathscr{D} \subset T \hat{X}$; thus a family of frames on patches of $\hat{X}$ related across patch overlaps by $G_{1}$ defines a distribution $\mathscr{D}$ and vice versa.

On $\mathbf{C}^{1 \mid 1}$ with its standard coordinates we introduce the standard almostsuperconformal structure:

$$
\hat{E}_{z}=\partial_{u} ; \quad \hat{E}_{+} \equiv D=\partial_{\theta}+\theta \partial_{u} .
$$

Even though the brackets $\hat{t}_{A B}{ }^{C}$ of this frame are not identically zero, still they are constants. Thus we will call this frame, and any other reduction locally equivalent to it, flat. Alternately, we say that a flat reduction defines a "superconformal structure," omitting the prefix "almost." Any set of local coordinates $u, \theta$ for which (3.5) define a superconformal structure will be called "superconformal coordinates" for that structure. Two different sets of superconformal coordinates for the same superconformal structure are said to differ by a "superconformal diffeomorphism," much as two sets of holomorphic coordinates differ by a holomorphic diffeomorphism [4].

From the previous discussion any flat frame must satisfy the necessary condition that its $t_{A B}{ }^{C}$ matches the $G_{1}$-invariant components of $\hat{t}_{A B}{ }^{C}$. This yields the conditions (3.3) as well as

$$
t_{+\bar{z}}^{z}=t_{+-}^{z}=0
$$

In addition the nondegeneracy condition implies that $t_{++}{ }^{z}$ must be nowherevanishing:

$$
t_{++}{ }^{z} \neq 0 \text {. }
$$

This in turn can be used to show that half of each of the conditions (3.3), (3.6) are actually redundant, related to the others by Jacobi identities via the definition (2.14). For example $t_{+\bar{z}} z=0$ follows when we use

$$
\left[E_{+},\left[E_{-}, E_{-}\right]\right]+2\left[E_{-},\left[E_{+}, E_{-}\right]\right]=0 .
$$

If a reduction defines a distribution $\mathscr{D}$ which is everywhere equivalent to the standard one up to first derivatives, then in particular $\mathscr{D}$ is holomorphic; thus we recover the definition of [26] from (3.3) and (3.6). Conversely a complex supermanifold $\hat{X}$ with holomorphic distribution $\mathscr{D}$ yields a family of frames: take $E_{+}$to be a holomorphic section of $\mathscr{D}$ and $E_{z}$ some holomorphic vector field everywhere linearly independent of $E_{+}$. With this choice (3.6) is clearly satisfied. It remains to be shown, however, that our definition agrees with the supergravity definition. In particular the latter approach makes extensive use of connections, curvature, and so on, none of which have yet appeared. 
B. Comparison to $2 d$ Supergravity. Before discussing supergravity per se, we note that an almost-superconformal structure can always be further reduced from the group $G_{1}$ (see (3.4)) to the smaller group

$$
G=\left\{\left(\begin{array}{cc}
B^{2} & \Gamma \\
0 & B
\end{array}\right) ; \quad B \text { invertible }\right\} \subseteq G_{1}
$$

by virtue of the nondegeneracy condition (3.7). That is, since $t_{++} z$ is nonvanishing we can always rescale $E_{z}$ to ensure that

$$
t_{++}^{z}=2 \text {. }
$$

$G$ is then the residual group of transformations preserving the "gauge condition" (3.10). Since the rescaling was canonical, the new $G$-structure contains no more information than the old $G_{1}$-structure. We will call either one an almost superconformal structure.

There is another gauge-fixing condition one can always impose without any additional choices. Consider the condition

$$
2 t_{+-}^{-}+t_{++}^{+}=0 .
$$

(We will motivate this condition in a moment.) Given a frame not satisfying (3.11) we can always uniquely obtain one which does by a $G$ transformation of the form $\left(\begin{array}{ll}1 & \Gamma \\ 0 & 1\end{array}\right)$. Given a frame which does satisfy it we get other such frames only when we apply transformations of the form

$$
\left(\begin{array}{cc}
e^{2(w+i l)} & 2 e^{2(w+i l)} \partial_{+} w \\
0 & e^{w+i l}
\end{array}\right)
$$

where $\partial_{+}$is $E_{+}$regarded as a differential operator. The transformations (3.12) are readily seen to be the $U(1)$ and super-Weyl transformations studied in [9]. Thus it seems likely that the usual supergravity approach to SRS will be just a partially gauge-fixed version of the discussion in the previous subsection. To verify this expectation we will now discuss those torsion constraints in $[8,9]$ which have not yet appeared in (3.3), (3.6), (3.10), (3.11).

Recall that in the supergravity approach one augments a choice of frame $E_{A}$ by an additional choice, that of a $U(1)$-structure with a connection. Thus we suppose that $E_{A}$ agree across patch boundaries not just up to transformations of the form (3.12), but in fact up to its $U(1)$ subgroup, and we introduce a connection 1-form $\phi_{A}$ with appropriate transformations. (A different frame in the same superconformal class will in general define a different connection, just as two conformal metrics on a Riemann surface define different connections $\nabla_{z}$.) We then define the torsion and curvature by

$$
\begin{aligned}
\nabla_{A} & =\partial_{A}+\phi_{A} M, \\
{\left[\nabla_{A}, \nabla_{B}\right] } & =-T_{A B}{ }^{C} \nabla_{C}+R_{A B} M .
\end{aligned}
$$

Here $M$ is the generator of $U(1)$; it equals $+i$ on tensors with one upper $z$ index and so on. A convenient choice of torsion constraints then 


$$
T_{a b}{ }^{c}=T_{\alpha \beta}{ }^{\gamma}=T_{+-}{ }^{z}=T_{--}{ }^{z}=0 ; \quad T_{++}{ }^{z}=-2,
$$

together with the conjugate conditions.

To compare (3.13) to our constraints, we first note that from the definitions

$$
t_{A B}{ }^{C}=-T_{A B}{ }^{C}+2 \phi_{[A} M_{B]}{ }^{C} .
$$

Thus of the constraints (3.13), some do not involve the connection at all, namely $0=t_{++}{ }^{-}=t_{+-}{ }^{z}=t_{--^{z}}{ }^{z}=t_{++}{ }^{z}=2 t_{+-}{ }^{-}+t_{++}{ }^{+}=0$ and their conjugates. Each of these conditions has already appeared, either as a genuine integrability condition (Eqs. (3.3), (3.6)) or as a gauge choice (Eqs. (3.10), (3.11)). The other two conditions

$$
T_{z \bar{z}}^{z}=T_{++}^{+}=0
$$

can simply be solved for $\phi_{z}$ and $\phi_{+}$, respectively. They serve the same role as the usual no-torsion condition $T_{z \bar{z}}{ }^{z}$ of Riemannian geometry: given a reduction all the way down to $S O(2)$ one obtains a unique connection by imposing (3.14).

Thus one can recover the full torsion constraints and the residual symmetry group of $2 \mathrm{~d}$ supergravity from the superconformal approach. Conversely all of the conditions in (3.3), (3.6) not yet mentioned follow from (3.13) via the Jacobi identities. The present approach makes it clear, however, that not all of the torsion constraints are on an equal footing. The integrability constraints (3.3), (3.6), and (3.7) will prove to be essential in the construction of an analog of the Cauchy-Riemann operator. The others are inessential (or conventional [28]), in that they fix things which don't need to be fixed in order to define string actions.

As mentioned, some of (3.3), (3.6) are redundant. Furthermore, if one has a reduction not satisfying these conditions it seems to be possible to modify it in a standard way so that it does [29]. This fact may be a consequence of the rigidity property discussed in Sect. 6.

There are sometimes reasons to prefer the superconformal approach over supergravity. In the latter approach one reduces all the way to $U(1)$, choosing a metric $E^{z} \otimes E^{\bar{z}}+E^{+} \otimes E^{-}$, only to write down conformally invariant actions. Similarly one obtains a connection, which however does not figure in the action. On the other hand, the entirely analytic approach of [4] is not always the most convenient either. Sometimes it is easier to deal not will holomorphic families of surfaces, bundles, and so on, but rather with equivalent families of frames, connections, etc. on fixed smooth spaces. The present approach interpolates between these two.

Whether we begin with (3.3), (3.6) or with (3.13), the conditions we arrive at are necessary conditions for the flatness of an almost-superconformal structure. To see that a reduction which is everywhere equivalent to the standard reduction (3.5) to first order is flat, or equivalent to (3.5) to all orders, one needs another integrability theorem. One can prove such a result by first passing to a Wess-Zumino gauge [9], but in the next section we give a proof directly from the integrability conditions. One reason for doing this is that for complex supermanifolds the proof given here generalizes easily to any number of dimensions. 


\section{Complex and Conformal Integrability}

We have already seen that the torsion constraints (3.3) and (3.6) are necessary conditions for the integrability of the complex and superconformal structures, respectively, i.e. for the existence of local complex or superconformal coordinates. In this section we turn to the task of proving the converse: the conditions (3.3) and (3.6) are sufficient to guarantee the integrability of the complex and superconformal structures. This result involves the super extension of the Frobenius theorem, with a few modifications of the classical proof. The follwing discussion of integrability roughly follows the approach in [30]. ${ }^{5}$

Integrability is a local question. Once we have covered $\hat{X}$ by patches with holomorphic coordinates in each patch, it follows by definition that across patches our coordinates will be related by holomorphic diffeomorphisms of $\mathbf{C}^{1 / 1}$, since the complex structure itself is globally defined. Similarly a superconformal structure will give an atlas of coordinate charts related by superconformal diffeomorphisms. On each patch we can take $\hat{X}$ to be split; moreover any parametrized family of $\hat{X}$ is locally a cartesian product. Thus without loss of generality we will work on a neighborhood $\hat{U} \subset \mathbf{R}^{2 \mid 2}$, or more precisely the product $\hat{Z} \times \hat{U}$ of $\hat{U}$ with some parameter space. We will usually suppress $\hat{Z}$ from the notation. A neighborhood $\hat{U}$ is of the form $\left(U, \mathscr{A}(U) \otimes \wedge\left(\mathbf{R}^{2}\right)\right)$, where $U \subset X$ is an ordinary open set and $\mathscr{A}(U)$ are the smooth ordinary functions on $U$.

A. Complex Integrability. We will work in stages, first discussing complex integrability. Specifically, suppose that $\left\{E_{z}, E_{+}\right\}$satisfy the complex torsion constraints (3.3), i.e. the commutators $\left[E_{z}, E_{+}\right]$and $\left[E_{+}, E_{+}\right]$don't contain any $E_{\bar{z}}$ or $E_{-}$ pieces. $\left\{E_{z}, E_{+}\right\}$therefore are said to define an integrable distribution $\Gamma$ in the complexified tangent space of $\hat{X}$. We will show that this implies the existence of coordinates $u, \theta$ such that $\partial / \partial u, \partial / \partial \theta$ span $\Gamma$.

The first step in the proof is to show that there exist complex vectors $Y, \Psi$ (generically called $Y_{(I)}$ ) spanning $\Gamma$, such that $\left[Y_{(I)}, Y_{(J)}\right]=0$. Let $P$ be a point in $U$ and $v$ a complex coordinate centered at $P$. We can then pick complex coordinates $\left\{v^{I}\right\} \equiv\{v, \bar{v}, \eta, \bar{\eta}\}$ on $\hat{U}$ such that at the point $P, \Gamma$ is spanned by $\left\{\partial_{v}, \partial_{\eta}\right\}$. This statement means that the given vectors $E_{+}, E_{z}$ take the form

$$
E_{z}=a \partial_{v}+\beta \partial_{\eta}, \quad E_{+}=\alpha \partial_{v}+b \partial_{\eta},
$$

when we set $v$ and $\eta$ to zero. $a$ and $b$ are nonzero constants. Equation (4.1) can always be arranged by a linear transformation on the coordinates $v^{I}$.

The coordinates $v, \eta$ define a map $c$ from $\hat{U}$ to $\mathbf{C}^{111}$. Furthermore, near $v=0$, $c_{*}$ takes the subspace $\Gamma$ isomorphically to $T^{1,0}\left(\mathbf{C}^{1 \mid 1}\right)$, since (4.1) remains invertible even when $v, \eta$ are not zero. Let $I=\left.c_{*}\right|_{\Gamma}$, the restriction of $c_{*}$ to $\Gamma$ on a neighborhood $\hat{U}^{\prime} \subset \hat{U}$ where it is invertible. Then on $\hat{U}^{\prime}$ one can define

$$
Y=I^{-1}\left(\partial_{v}\right) ; \quad \Psi=I^{-1}\left(\partial_{\eta}\right) .
$$

It is then easy to check that

\footnotetext{
${ }^{5}$ Another discussion of integrability appears in [31]
} 


$$
c_{*}\left(\left[Y_{(I)}, Y_{(J)}\right]\right)=\left[\frac{\partial}{\partial v^{I}}, \frac{\partial}{\partial v^{J}}\right]=0 .
$$

The condition for complex integrability says that $\left[Y_{(I)}, Y_{(J)}\right]$ itself lies in $\Gamma$; therefore since $I$ is one-to-one, we conclude that

$$
\left[Y_{(I)}, Y_{(J)}\right]=0 .
$$

We have thus constructed a commuting set of vector fields which span $\Gamma$.

The existence of vectors $Y_{(I)}$ satisfying (4.4) will allow us to define the desired complex coordinates by a standard procedure. However, first we must check that a single vector field can be integrated to provided a flow, i.e. given a vector $Y$ on $\mathbf{R}^{2 \mid 2}$ (say), we want to find a family of maps $A(y): \mathbf{R}^{2 \mid 2} \rightarrow \mathbf{R}^{2 \mid 2}$ such that

$$
\frac{\partial A}{\partial y}=Y[A(y)], \quad A(0)=\text { identity }
$$

In the case where $Y$ is an even vector, we show integrability order by order. Write $A\left(v^{I} ; y\right)=\left(Z\left(v^{I} ; y\right), \Xi\left(v^{I} ; y\right)\right)$ and $Y^{I}\left(v^{I} ; y\right)=\left(Y^{v}, \overline{Y^{v}}, Y^{\eta}, \overline{Y^{\eta}}\right)$. If we have not a single vector field but a family of them with $l-2$ odd parameters $\zeta^{i}$, we simply lump the $\zeta^{i}$ with $\eta, \bar{\eta}$ and consider flows on $\mathbf{R}^{2 \mid l}$ with most of the components of $Y^{I}$ equal to zero. To accommodate this case we therefore consider any number of $\eta^{i}$ and $\Xi^{i}$. Expanding (4.5) in the $\eta^{i}$ gives $2^{l}$ coupled first-order differential equations, which we can interpret as defining the flow of a single ordinary vector field in a space of dimension $2^{l}$. This problem has a unique solution by a classical theorem of differential geometry [30].

The case of an odd vector field $\Psi$ is a bit tricker. Indeed, a necessary condition for a solution of

$$
\frac{\partial A}{\partial \psi}=\Psi[A(\psi)]
$$

to exist (for odd $\psi$ ) is clearly $[\Psi, \Psi]=0\left(\right.$ since $\left.(d / d \psi)^{2}=0\right)$. To see that this is also a sufficient condition, again let $Z, \Xi$ be the even and odd components of the map $A$. Then Eq. (4.6) becomes

$$
\alpha=\Psi^{v}(Z, \Xi) ; \quad a=\Psi^{\eta}(Z, \Xi)
$$

where $\alpha, a$ are defined by

$$
Z(v, \eta ; \psi)=v+\psi \alpha(v, \eta), \quad \Xi(v, \eta ; \psi)=\eta+\psi a(v, \eta) .
$$

To show that these equations have a solution, expand (4.7) in powers of $\psi$ and solve for $a, \alpha$, ignoring the terms proportional to $\psi$ on the right-hand side. Take the solution thus obtained and put it back into the $\psi$ terms. We immediately see that they vanish, and therefore that the solution is consistent, if and only if $[\Psi, \Psi]=0$.

Application of this result now provides us with a definition of the desired complex coordinates. Define the families of diffeomorphisms $A_{(i)}: \hat{U} \times \mathbf{R} \rightarrow \mathbf{R}^{2 \mid 2}$, $i=1,2$ by 


$$
\frac{\partial A_{(1)}}{\partial u^{1}}=\operatorname{Re} Y ; \quad \frac{\partial A_{(2)}}{\partial u^{2}}=\operatorname{Im} Y,
$$

and similarly define diffeomorphisms $B_{(1)}, B_{(2)}$, parametrized by odd parameters $\theta^{1}, \theta^{2}$, by the equation

$$
\frac{\partial B_{(1)}}{\partial \theta^{1}}=\operatorname{Re} \Psi ; \quad \frac{\partial B_{(2)}}{\partial \theta^{2}}=\operatorname{Im} \Psi
$$

The vectors $Y, \Psi$ are the ones constructed in (4.2); in particular $[\Psi, \Psi]=0$. Geometrically $u^{1}, u^{2}, \theta^{1}$ and $\theta^{2}$ are the "time" of flow along the integral curves of the respective vector fields. Now define $\chi: \mathbf{R}^{2 \mid 2} \rightarrow \hat{U}$ by

$$
\chi\left(u^{1}, u^{2}, \theta^{1}, \theta^{2}\right)=\left[A_{(1)}\left(u^{1}\right) \circ A_{(2)}\left(u^{2}\right) \circ B_{(1)}\left(\theta^{1}\right) \circ B_{(2)}\left(\theta^{2}\right)\right](v=0, \eta=0) .
$$

$\chi$ defines new coordinates for $\hat{U}$ by

$$
\left\{y^{M}\right\}=\left(u^{1}, u^{2}, \theta^{1}, \theta^{2}\right)=\chi^{-1} .
$$

Let $\tilde{Y}_{(M)}$ denote the real vector $\left(\frac{1}{2} \operatorname{Re} Y,-\frac{1}{2} \operatorname{Im} Y, \frac{1}{2} \operatorname{Re} \Psi,-\frac{1}{2} \operatorname{Im} \Psi\right)$. Then the relation (4.4) trivially implies the relation $\left[\tilde{Y}_{(M)}, \tilde{Y}_{(N)}\right]=0$. Further, it can easily be checked that the vectors $\chi_{*}\left(\partial / \partial y^{M}\right)$ are nondegenerate near $P$. These two conditions are sufficient to ensure that the coordinates $y^{M}$ are well defined; it is also easy to see that $\tilde{Y}_{(M)}=\frac{1}{2} \partial / \partial y^{M}$.

To sum up, we have shown that there exist coordinates $u=u^{1}+i u^{2}, \theta=\theta^{1}+i \theta^{2}$ such that

$$
Y=\partial_{u}, \quad \Psi=\partial_{\theta}
$$

span the distribution $\Gamma$. The frame $\left\{E_{z}, E_{+}\right\}$then has an expansion

$$
E_{z}=b \partial_{u}+\beta \hat{\partial}_{\theta}, \quad E_{+}=\gamma \partial_{u}+c \partial_{\theta},
$$

where $b, c$ are nonvanishing near $P$. Note that in general $b, \beta, c, \gamma$ will not themselves be holomorphic functions. The point is that there exists a local $G L(1 \mid 1, \mathbf{C})$ transformation of $E_{A}$ taking (4.12) to an equivalent frame (4.11).

B. Conformal Integrability. Once we have a complex structure, we can go one step further and inquire as to whether the full torsion constraints (3.3), (3.6), (3.7) are sufficient to provide a superconformal structure. The important constraints at this stage are $t_{+-}{ }^{z}=t_{+-}{ }^{\bar{z}}=0$, i.e.

$$
\left[E_{+}, E_{-}\right]=\varepsilon E_{+}+\bar{\varepsilon} E_{-} .
$$

(Recall that these torsion constraints, combined with the complex structure constraints (3.3), imply the other conformal constraint in (3.6) via the Jacobi identity (3.8).)

The proof of the integrability of such an almost-superconformal structure follows the same basic strategy as that used to prove complex integrability. As before, work on a coordinate neighborhood $\hat{U}$, where $E_{+}$takes the form in (4.12) and $E_{-}$is given by the complex conjugate equation. Once again we work near a point $P \in U$; we take $P$ to have coordinate $u=0$. Setting $u=\theta=0, E_{+}$has the form

$$
E_{+}=\gamma_{0} \partial_{u}+c_{0} \partial_{\theta} \equiv f_{+},
$$


where $c_{0}, \gamma_{0}$ are some constants. The reduced vector field $f_{+}$extends to a "constant" vector field on the whole open set $\hat{U}$, and if we let $f_{-}$be its complex conjugate we get $\left[f_{+}, f_{-}\right]=0$. Let $E_{+} \equiv \kappa \partial_{u}+k f_{+}$define functions $k$ and $\kappa$ on $\hat{U}$. For an arbitrary vector $\alpha \partial_{u}+a f_{+}$we define a projection $\Pi$ :

$$
\Pi\left(\alpha \partial_{u}+a f_{+}\right) \equiv a f_{+},
$$

together with the c.c. relation. If we define $\mathscr{D}$ to be the subspace of the tangent space spanned by $E_{+}$, then clearly

$$
\left.\pi \equiv \Pi\right|_{\mathscr{L}}: \mathscr{D} \rightarrow \operatorname{span}\left\{f_{+}\right\}
$$

is an isomorphism in some neighborhood $\hat{U}^{\prime} \subset \hat{U}$. Now let $F_{+}=\pi^{-1}\left(f_{+}\right)$. Then $F_{+}$takes the form

$$
F_{+}=f_{+}+\delta \partial_{u}
$$

it satisfies the same nondegeneracy condition (3.7) as $E_{+}$. It is once again easy to check that

$$
\pi\left[F_{+}, F_{-}\right]=\left[f_{+}, f_{-}\right]=0,
$$

where $F_{-}$is the conjugate of $F_{+}$. However, the fact that $F_{+}$lies in $\mathscr{D}$ together with the constraint (4.13) implies

$$
\left[F_{+}, F_{-}\right]=\phi F_{+}+\bar{\phi} F_{-} .
$$

Since $\pi$ is one-to-one on $\mathscr{D} \oplus \overline{\mathscr{D}}$, (4.18) implies

$$
\left[F_{+}, F_{-}\right]=0 \text {. }
$$

From this last equation we see that $F_{+}$(respectively $F_{-}$) varies analytically (anti-analytically); indeed, since $F_{+}, F_{-}$look like $F_{+}=m \partial_{\theta}+\mu \partial_{u}, F_{-}=\bar{m} \partial_{\bar{\theta}}+\bar{\mu} \partial_{\hat{u}}$, the conditions (4.20) and (3.7) yield the statement that $m, \mu$ are analytic. To put the frame in the canonical form (3.5), we now just follow the argument sketched in [6]. We rewrite $F_{+}$as $F_{+}=n(u, \theta)\left(\partial_{\theta}+v(u, \theta) \partial_{u}\right)$, and then introduce new coordinates $\theta, u^{\prime}(u, \theta)$ so that $\partial_{\theta}+v(u, \theta) \partial_{u}=\partial_{\theta}+\theta \partial_{u^{\prime}}$. It is easily checked that it is always possible to find such a $u^{\prime}$, and thus we have conformal integrability.

We conclude by stating the general result on integrability:

Result. Given a frame $E_{A}$ satisfying the torsion constraints (3.3) (the complex torsion constraints), there exist complex coordinates $\tilde{u}, \tilde{\theta}$ near any point of $X$ such that $\partial_{\tilde{u}}, \partial_{\tilde{\theta}}$ everywhere span the same space as $E_{z}, E_{+}$. Thus the almost-complex structure specified by $E_{A}$ is locally equivalent to the standard complex structure on $\mathbf{C}^{111}$. Furthermore, if the frame $E_{A}$ also satisfies the torsion constraints (3.5), (3.7) (the superconformal torsion constraints), then we can choose new holomorphic coordinates $u, \theta$ such that $\partial / \partial \theta+\theta(\partial / \partial u)$ spans the same space $\mathscr{D}$ as $E_{+}$. In this case the almost-superconformal structure specified by $E_{A}$ (see (3.4)) is locally equivalent to the standard flat superconformal structure on $\mathbf{C}^{1 / 1}$.

\section{An Important Sequence}

A key feature of ordinary Riemann surfaces is the fact that the holomorphic tangent $\mathscr{T}$ has just one complex dimension. This observation enables us to write down a 
differential operator with values in a line bundle, namely the dual $\omega=\mathscr{T}^{*}$ to $\mathscr{T}$. We simply choose any nonvanishing local section $e_{z}$, and define

$$
\partial=e^{z} \otimes \partial_{z},
$$

where $\partial_{z}$ is $e_{z}$ regarded as a complex differential operator. Since $\mathscr{T}$ is onedimensional it doesn't matter which section $e_{z}$ we choose.

For a complex manifold of dimension greater than one, (5.1) has no immediate analog. If $X$ is equipped with a one-dimensional distribution $\mathscr{D}$, then we can follow this same recipe to get a differential operator with values in the dual $\mathscr{D}^{*}$. Such a $\partial$, however, lacks two other key features of (5.1), features which are central to its usefulness:

- The operator $\partial$ takes its values in a bundle $\omega$ which can be regarded as a square root of the bundle of volume forms: vol $\cong \omega \otimes \bar{\omega}$. Thus it can be used to define a quadratic functional on the set of functions on $X$, the "matter action"

$$
S[f]=\int_{X} \partial f \bar{\partial} f
$$

- The operator $\bar{\partial}$ forms a short exact sequence:

$$
0 \rightarrow \mathcal{O} \subset \mathscr{A} \stackrel{\overline{\hat{o}}}{\rightarrow} \Omega^{0,1} \rightarrow 0 .
$$

Here $\mathcal{O}$ are the local holomorphic functions, $\mathscr{A}$ are the smooth ones, and $\Omega^{0,1}$ are smooth $(0,1)$-forms. The sequence (5.2) is called a "resolution of $\mathcal{O}$," and it forms the basis of the theory of DET line bundles.

Unfortunately neither of the above properties is generally valid in greater than one dimension. In particular, the half-volume forms are in general wedged from many covectors, not the dual of a single one. Nevertheless, in this section we will see that matters improve when we pass to SRS. We will recover both of the above properties; in this sense SRS behave as if they had just one dimension, even though the nonintegrability of $\mathscr{D}$ prevents us from finding a single good coordinate $\eta$ with $D=\partial / \partial \eta$.

To examine the first property, we note that volume forms are not simply top wedge products in the super category. Indeed, since $d \theta^{i} d \theta^{j}=d \theta^{j} d \theta^{i}$, wedge products never stop. Instead, define [17]

$$
\widehat{\mathrm{vol}}=\operatorname{DET}\left(T^{*} \hat{X}\right)
$$

the line bundle whose transition functions are the Berezin determinants of the

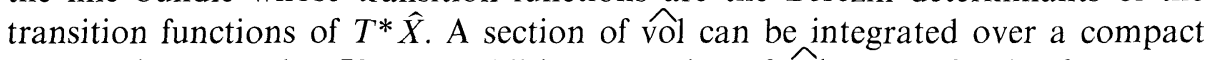

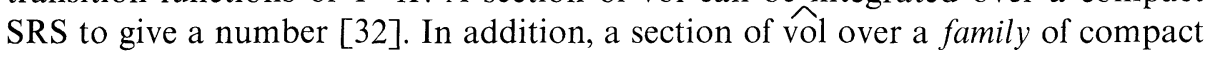
SRS can be integrated to give a function on the parameter space.

In the holomorphic case we can write $\widehat{\mathrm{vol}}=\hat{\omega} \otimes \hat{\bar{\omega}}$, where $\hat{\omega}=\operatorname{DET}\left(T^{*(1,0)} \hat{X}\right)$ is a holomorphic line bundle. The key observation needed to establish the first point above is that for SRS one has

$$
\hat{\omega} \cong \mathscr{D}^{*} .
$$


To prove this choose any local set of superconformal coordinates $u, \theta$, and set up the correspondence $[d u d \theta] \leftrightarrow D^{*}$. ( $D^{*}$ is the section of $\mathscr{D}^{*}$ dual to $D=\partial_{\theta}+\theta \partial_{u}$.) If any other choice $u^{\prime}, \theta^{\prime}$ results in the same correspondence, then we will have shown that (5.3) is canonical. But $u^{\prime}, \theta^{\prime}$ have the property that $D$ is proportional to $D^{\prime}=\partial_{\theta^{\prime}}+\theta^{\prime} \partial_{u^{\prime}}$. Reexpressing $D$ in terms of $u^{\prime}, \theta^{\prime}$ we get [4]

$$
D u^{\prime}=\theta^{\prime} D \theta^{\prime} \text {. }
$$

From this one shows that

$$
\begin{aligned}
\operatorname{det}\left(\begin{array}{ll}
\partial_{u} u^{\prime} & \partial_{u} \theta^{\prime} \\
\partial_{\theta} u^{\prime} & \partial_{\theta} \theta^{\prime}
\end{array}\right) & =\operatorname{det}\left[\left(\begin{array}{cc}
1 & 0 \\
-\theta & 1
\end{array}\right)\left(\begin{array}{cc}
\partial u^{\prime}+\theta^{\prime} \partial \theta^{\prime} & \partial \theta^{\prime} \\
0 & D \theta^{\prime}
\end{array}\right)\left(\begin{array}{cc}
1 & 0 \\
\theta^{\prime} & 1
\end{array}\right)\right] \\
& =\frac{\partial u^{\prime}+\theta^{\prime} \partial \theta^{\prime}}{D \theta^{\prime}}=D \theta^{\prime} .
\end{aligned}
$$

We have taken $D$ of (5.4) for the last step and abbreviated $\partial_{u}$ to $\partial$.

Thus $\left[d u^{\prime} d \theta^{\prime}\right]=\left(D \theta^{\prime}\right)[d u d \theta]$, while $D^{\prime}=\left(D \theta^{\prime}\right)^{-1} D$. This establishes the isomorphism (5.3), and with it the existence of an operator $\hat{\partial}$ on a SRS taking values in the half-volume forms. In local superconformal coordinates $\hat{\partial}$ simply looks like $[d u d \theta] \otimes D[4]$. In arbitrary holomorphic coordinates we can instead write [14]

$$
\hat{\partial}=[d u d \theta] \operatorname{det}\left(\begin{array}{cc}
E_{u}{ }^{z} & E_{u}{ }^{+} \\
E_{\theta}{ }^{z} & E_{\theta}{ }^{+}
\end{array}\right) \otimes \partial_{+},
$$

analogously to (5.1). Here $E^{A}$ is a reduction to $G$ (see (3.9)). Equation (5.5) is clearly coordinate-invariant. It is also $G$-invariant, since the Berezin determinant of the graded matrix in (3.9) is $B^{2}$. $B^{-1}=B$. The fact that inverses appear in Berezin determinants is the key difference between 2 and $1 / 1$ dimensions. It is this fact which lets a volume form transform as the dual to a vector field once a distribution $\mathscr{D}$ has been chosen.

We can now just verify the second point above. Once again, on an ordinary manifold of dimension greater than one the prospects for such a result appear dim. If a function is annihilated by one directional derivative, this usually says nothing about its other derivatives, and so it seems hard for (5.2) to be exact at $\mathscr{A}$. On a supermanifold, however, a single vector field can be nonintegrable, as is $D$. Thus locally imposing $\bar{D} f=0$ also imposes $\bar{D}(\bar{D} f)=\bar{\partial} f=0$, so that being in the kernel of $\hat{\bar{\partial}}$ indeed ensures that $f$ is holomorphic. Let $\hat{\mathscr{A}}^{0,1}$ denote the smooth sections of $\hat{\bar{\omega}}$, i.e. $\hat{\mathscr{A}}^{0,1} \equiv \hat{\bar{\omega}} \otimes \hat{\mathscr{A}}$. It is easy to show that every differential in $\hat{\mathscr{A}}^{0,1}$ is $\hat{\bar{\partial}}$ of something, and so we get a short exact sequence

$$
0 \rightarrow \hat{\mathcal{O}} \hookrightarrow \hat{\mathscr{A}} \stackrel{\hat{\overline{\hat{\theta}}}}{\rightarrow} \hat{\mathscr{A}}^{0,1} \rightarrow 0,
$$

the fundamental sequence of a SRS. Equation (5.6) plays the same role as the Dolbeault sequence on ordinary Riemann surfaces.

Before closing this section we now give another characterization of $\hat{\partial}$. Another intrinsically defined differential operator is the Dolbeault operator, $\bar{\partial}: \mathscr{A} \rightarrow \hat{\Omega}^{0,1}$, where $\hat{\Omega}^{p, q}$ are the smooth $(p, q)$-forms. $\partial$ can be defined using only the complex structure, not the full superconformal structure. However, $\hat{\Omega}^{0,1}$ is two-dimensional, 
and $\bar{\partial}$ defines a sequence with more than three entries,

$$
0 \rightarrow \hat{\mathcal{O}} \hookrightarrow \hat{\mathscr{A}} \stackrel{\overline{\hat{O}}}{\rightarrow} \hat{\Omega}^{0,1} \stackrel{\bar{\partial}}{\rightarrow} \hat{\Omega}^{0,2} \rightarrow \cdots
$$

We can truncate this sequence, however, if we replace $\hat{\Omega}^{0,1}$ by the sheaf of closed local $(0,1)$-forms $\mathscr{Z}^{0,1}$,

$$
0 \rightarrow \hat{\mathcal{O}} \hookrightarrow \hat{\mathscr{A}} \stackrel{\hat{0}}{\rightarrow} \mathscr{Z}^{0,1} \rightarrow 0
$$

So far this is nothing new. If $\hat{X}$ has a superconformal structure, however, we can canonically identify closed antiholomorphic forms with arbitrary smooth sections of the line bundle $\hat{\bar{\omega}}$ :

$$
\hat{\mathscr{A}}^{0,1} \cong \mathscr{Z}^{0,1} .
$$

Note that by itself $\mathscr{Z}^{0,1}$ is not the sheaf of sections of any bundle; its sections satisfy a differential equation. To verify (5.8), choose local superconformal coordinates and identify $\beta[d \bar{u} d \bar{\theta}]$ with $(d \bar{u}-d \bar{\theta} \cdot \bar{\theta}) \bar{D} \beta+d \bar{\theta} \beta$, where $\beta$ is any function. As before one shows that this identification factors through a change of coordinates.

We can identify $\hat{\bar{\partial}}$ with $\bar{\partial}$ under (5.8). Thus we again recover the fundamental sequence (5.6), from (5.7). A theorem about resolutions then implies that the cohomology of $\hat{\mathcal{O}}$ can be computed from the kernel and cokernel of $\hat{\bar{\partial}}$ [1]. Moreover, one can couple holomorphic line bundles to the sequence (5.6), leading into a theory of $\hat{\bar{\partial}}$ coupled to families of bundles as well as families of SRS. This theory let us define the action for supersymmetric first-order systems and the bundles where the resulting partition functions live. As in the classical case, line bundles are partially classified by an integer invariant, the Chern number. All bundles of vanishing Chern number can be represented by connections on the trivial bundle, but in contrast to the classical case, these connections are not necessarily flat [33].

\section{Moduli of SRS}

A fundamental result in Riemann surface theory states that the moduli space $\mathscr{M}$ of curves is itself a complex space [34]. We can think of this result in two ways. From a complex analytic point of view we can first define the notion of a holomorphic family of Riemann surfaces, roughly a projection

$$
\pi: W \rightarrow Z
$$

whose fibers are all Riemann surfaces.

Given such a family we can ask whether it contains "every" Riemann surface, and if so whether it contains each one "only once." More precisely, one can first ask a local question: near each point $Q$ of $Z$ does moving in $Z$ constitute the most general deformation of the Riemann surface $X_{0} \equiv \pi^{-1}(Q)$ ? To answer the question one defines the "Kodaira-Spencer map" associated to $\pi$ at $Q$. This map takes the space $T_{Q} Z$ of tangents to $Z$ to an abstract space of deformations of $X_{0}$. If it is everywhere an isomorphism, then locally $\pi: W \rightarrow Z$ describes a general deformation with no redundant parameters. 
In general no good family (6.1) of deformations of a complex manifold will exist. For the case of Riemann surfaces, however, such a family exists and is moreover universal, in the sense that any other family can be built from it. The parameter space $Z$ of their universal family is called the moduli space of Riemann surfaces, and is denoted by $\mathscr{M}$. It is by construction a complex space. An analysis of SRS along these lines has been carried out in $[5,6]$; again one finds a universal holomorphic family of SRS with parameter space the supermoduli space $\hat{\mathscr{M}}$.

A. "Classical" Approach. There is a more classical approach to the moduli problem, however, one which is more in line with the differential-geometric viewpoint of this paper. This approach sometimes has practical advantages over the more abstract Kodaira-Spencer treatment, and in any case it seems more familiar to string theorists. Accordingly in this section we will briefly describe some basic facts about $\hat{\mathscr{M}}$ in the present language. In particular we will discuss the related space $\hat{\mathscr{M}}_{c}$ of complex $1 \mid 1$-dimensional curves, and its relation to $\hat{\mathscr{M}}$.

To begin, we can think of defining ordinary moduli space $\mathscr{M}$ as simply the collection of all Riemann surfaces. More precisely we call two Riemann surfaces equivalent if there exists a holomorphic diffeomorphism between them [34]. $\mathscr{M}$ then becomes the space of all inequivalent complex structures $J$ on a fixed 2-surface (see Sect. 1), with a topology induced from the function space of all $J$. We can at once build a family of Riemann surfaces over $\mathscr{M}$, and this time the family includes every surface exactly once by definition. What is now not clear is whether the family thus obtained is holomorphic, nor even whether $\mathscr{M}$ is a complex space.

To show that $\mathscr{M}$ is complex one can equip it with an almost-complex structure, show that the latter is integrable, and invoke the integrability theorem. Similarly one can define the space $\hat{\mathscr{M}}_{c}$ of complex curves as the space of integrable complex structures $J$ on a fixed surface $X_{r}$ of $2 \mid 2$ real dimensions, equip it with a natural almost-complex structure, and verify integrability, as done in [27]. The result of Sect. 4A, suitably generalized to several dimensions, then shows that $\hat{\mathscr{M}}_{c}$ is a complex space. To turn this into a result about the moduli space $\hat{\mathscr{M}}$ of SRS, we will now show that $\hat{\mathscr{M}}$ is embedded in $\hat{\mathscr{M}}_{c}$.

$\hat{\mathscr{M}}_{\mathrm{c}}$ consists of integrable reductions of $X_{\mathrm{r}}$ to $G L(1 \mid 1, \mathrm{C}) ; \hat{\mathscr{M}}$ consists of integrable reductions to the smaller subgroup $G_{1}$ of upper-triangular matrices. Since the former are a special case of the latter, we get a natural "forgetful" map $\phi: \hat{\mathscr{M}} \rightarrow \hat{\mathscr{M}}_{c}$. However, $\phi$ need be neither one-to-one nor onto. Some complex manifolds may admit no holomorphic distribution $\mathscr{D}$ as in Sect. 3; others could in principle admit many different choices. What we will see is that the former possibility is realized, while the latter is not: locally, at least, superconformal structures are rigid in their complex class.

We will work near a given SRS $\hat{X}$ and examine the possible deformations of its complex structure $J$. Recall that evey supermanifold contains an embedded copy of its body as the "locus where the nilpotents are zero" (see (2.7)). In the case of $\hat{\mathscr{M}}$, the body is spinmoduli space $\mathscr{M}_{\text {spin }}$ and its image is called the "split locus" for reasons mentioned at the beginning of Sect. 3: each SRS described by a point of $\mathscr{M}_{\text {spin }}$ is in fact split. For simplicity we will initially take $\hat{X}$ to be on the split locus, generalizing later to all of $\hat{\mathscr{M}}$. 
Choose superconformal coordinates for $\hat{X}$, so that locally $J$ takes the form ${ }^{6}[27]$

$$
\begin{aligned}
J & =i\left(E^{z} \otimes E_{z}+E^{+} \otimes E_{+}\right)+\text {c.c } \\
& =i\left[(d u-d \theta \cdot \theta) \otimes \partial_{u}+d \theta \otimes\left(\partial_{\theta}+\theta \partial_{u}\right)\right]+\text { c.c. } \\
& =i\left[d u \otimes \partial_{u}+d \theta \otimes \partial_{\theta}\right]+\text { c.c. } .
\end{aligned}
$$

$J$ is well defined across patch boundaries, it satisfies $J^{2}=-1$ and it defines $T^{*(1,0)} \hat{X}=\operatorname{span}\{d u, d \theta\}$, which is integrable. Let $J^{\prime}=J+i \Delta$ be a nearby complex structure. Then we have

$$
J \Delta+\Delta J=0
$$

so the components of $\Delta$ are

$$
[\Delta] \equiv\left(\begin{array}{ccc}
0 & \text { c.c. } \\
j_{\bar{u}}{ }^{u} & \gamma_{\bar{u}}{ }^{\theta} & \\
\beta_{\bar{\theta}}{ }^{u} & k_{\bar{\theta}}{ }^{\theta} & 0
\end{array}\right) .
$$

Most of the information in $\Delta$ does not represent true deformations in $\hat{\mathscr{M}}_{c}$. For example, the Lie derivative of $J$ along any real vector field $Y$ describes a $\Delta$ corresponding to a diffeomorphism, and hence a trivial change in $\hat{\mathscr{M}}_{c}$. In the case of ordinary Riemann surfaces we have

$$
\begin{aligned}
\Delta & =\left(\begin{array}{cc}
0 & j_{u}^{\bar{u}} \\
j_{\bar{u}} & 0
\end{array}\right), \\
\Delta & =-i \mathscr{L}_{Y} J=2\left[\partial_{\bar{u}} Y^{u} \otimes \partial_{u}-\partial_{u} Y^{\bar{u}} \otimes \partial_{\bar{u}}\right], \\
j_{\bar{u}}{ }^{u} & =2 \partial_{\bar{u}} Y^{u} .
\end{aligned}
$$

What is left when we delete all the trivial changes (6.3)? The obstruction to solving (6.3) for $Y$ given an arbitrary $j_{u}^{\bar{u}}$ is the Dolbeault group $H_{\bar{\partial}}^{-1,1}(X)$. By the Dolbeault theorem, this is the same as $H^{1}\left(\omega^{*}\right)$, where $\omega^{*}$ is the holomorphic tangent to $X$. By Serre duality, the latter is in turn dual to $H^{0}\left(\omega^{2}\right)$, and we recover the familiar result that the cotangent to $\mathscr{M}$ is the space of quadratic differentials [34].

In the super case things are almost as straightforward at the split locus. At first it seems as though the four real components of a vector field $Y$ are insufficient to eliminate most of the eight degrees of freedom in (6.2). We still have not imposed the integrability conditions (3.3), however. Solve to first order for the $+i$ eigenvectors $\eta^{z}, \eta^{+}$of $J^{\prime}$. Expand $d \eta^{z}, d \eta^{+}$in terms of $\eta^{z}, \eta^{+}$and their conjugates and set the terms with $\eta^{\bar{z}}, \eta^{-}$to zero to get the four equations, ${ }^{7}$

$$
\begin{array}{ll}
\partial_{\bar{\theta}} \beta=0 ; & \partial_{\bar{\theta}} j=\partial_{\bar{u}} \beta ; \\
\partial_{\bar{\theta}} k=0 ; & \partial_{\bar{\theta}} \gamma=\partial_{\bar{u}} k .
\end{array}
$$

Now we can subject $J$ to a diffeomorphism as before, finding

${ }^{6}$ This $J$ is actually the dual to the object considered earlier; it maps $T^{*} \hat{X}$ to itself

7 Since the exterior derivative is dual to the Lie bracket these equations are just a restatement of (3.3) 


$$
j_{\bar{u}}{ }^{u}=2 \partial_{\bar{u}} Y^{u} ; \quad \gamma_{\bar{u}}{ }^{\theta}=2 \partial_{\bar{u}} Y^{\theta} ; \quad \beta_{\bar{\theta}}{ }^{u}=2 \partial_{\bar{\theta}} Y^{u} ; \quad k_{\bar{\theta}}{ }^{\theta}=2 \partial_{\bar{\theta}} Y^{\theta} .
$$

Note that a holomorphic vector field $Y$ trivially leaves $J$ unchanged. Also (6.5) automatically satisfy (6.4), as they must since they don't affect the complex structure at all.

Now we can ask when a perturbation is both legitimate (satisfies (6.4)) and nontrivial (not of the form (6.5)). That is, given $j, \gamma, \beta, k$ we ask how much of the information can be removed by adding something of the form (6.5). Expanding $j$ as $j=j_{1}+\theta j_{2}+\bar{\theta} j_{3}+|\theta|^{2} j_{4}$ etc., we find that the obstruction to removing $j_{1 \bar{u}}{ }^{u}{ }{ }^{2}$ again $H_{\bar{\partial}}^{-1,1}(X)$. For both $j_{2 \bar{u}}{ }^{\theta}$ and $\gamma_{1 \bar{u}}{ }^{\theta}$ the obstruction is $H_{\bar{\partial}}^{-1 / 2,1}(X)$; for $\gamma_{2 \bar{u}}$ it is $H_{\overline{\mathrm{o}}}^{0,1}(X)$. All told, using the Riemann-Roch theorem we find $4 g-3$ independent even deformations and $4 g-4$ odd ones. The dimension of $\hat{\mathscr{M}}_{c}$ is thus [35] $4 g-3 \mid 4 g-4$.

Since supermoduli space is known to have dimension $3 g-3 \mid 2 g-2$, we at once see that certain deformations of $\hat{X}$ admit no superconformal structure. However, one can easily show that every nontrivial deformation of a SRS is still nontrivial when regarded as a deformation of a complex curve: simply take the variation of such a family (e.g. the one described in [16]) and show that it cannot be written in the form (6.5). Thus we have that $\hat{\mathscr{M}}$ is embedded in $\hat{\mathscr{M}}_{c}$, at least near the split locus.

B. Analytic Treatment. To get a more general result we should look for a proof which does not rely on special coordinate systems. For this we will employ the analytic approach mentioned at the beginning of this section.

A Riemann surface can be thought of as patches of $\mathbf{C}$ with transition functions $g_{\alpha \beta}$ respecting the holomorphic structure. To deform the surface we can deform each patching function, replacing it by a new one, $g_{\alpha \beta}^{\prime}$ again respecting the complex structure of $\mathbf{C}$. The new and old are said to differ by a local automorphism of the standard complex structure on $\mathbf{C}$. The generators of such automorphisms are clearly just the holomorphic vector fields, local sections of the sheaf $\mathscr{T}$. Requiring that $g_{\alpha \beta}^{\prime}$ continue to satisfy the cocycle condition, and that it not be related to $g_{\alpha \beta}$ by a mere change of local trivializations, leads by a standard argument to the identification $^{8}$

$$
T^{1,0} \mathscr{M} \cong H^{1}(\mathscr{T}) .
$$

Since $\mathscr{T} \cong \omega^{*}$ for a Riemann surface, we again recover the result about quadratic differentials from Serre duality.

For complex 1|1 manifolds the situation is much the same. Holomorphic vector fields again generate automorphisms of the complex structure on patch overlaps, and so we get

$$
T^{1,0} \hat{\mathscr{M}}_{\mathrm{c}} \cong H^{1}(\hat{\mathscr{T}}) .
$$

For SRS, however, the automorphisms must preserve the full superconformal structure. The infinitesimal analog of (5.4) says that local automorphisms are

${ }^{8}$ More precisely, $\mathscr{T}_{\mathscr{M}}=R^{1} \pi_{*}\left(\mathscr{T}_{W / Z}\right)$ 
generated by

$$
\text { aut } \equiv\left\{v=a \partial_{u}+\frac{1}{2}(D a) D\right\} .
$$

We have chosen superconformal coordinates, but in fact aut is intrinsically defined. ${ }^{9}$ It is clear from (6.6) that we have $\hat{\mathscr{T}}=$ aut $\oplus \mathscr{D}$, and so

$$
T^{1,0} \hat{\mathscr{M}}_{c} \cong T^{1,0} \hat{\mathscr{M}} \oplus H^{1}(\mathscr{D}) \text {. }
$$

Thus the holomorphic tangent to moduli space everywhere sits naturally in that of $\hat{\mathscr{M}}_{c}$, generalizing the previous subsection. Moreover, at the split locus we have $\mathscr{D} \cong \omega^{-1 / 2} \oplus \mathcal{O}$, so that $H^{1}(\mathscr{D})$ indeed supplies the extra $g \mid 2 g-2$ dimensions of $\hat{\mathscr{M}}_{c}$ missing from $\hat{\mathscr{M}}$.

Since $\hat{\mathscr{M}}$ sits holomorphically inside $\hat{\mathscr{M}}_{c}$ while the latter is a complex space [27], so is $\hat{\mathscr{M}}$. Alternately one can simply construct complex coordinates for $\hat{\mathscr{M}}$ which everywhere respect the natural almost complex structure induced from $\hat{\mathscr{M}}_{c}$; this is done in $[5,16]$.

\section{Conclusion}

In this paper we have described some of the foundations of super Riemann surface theory. Further developments regarding line bundles on SRS will appear in [33]. Much work remains to be done, both in the direction of formal developments of the tools and techniques of SRS and in the direction of application of these tools to string theory.

Many challenging questions remains about supermoduli. It is not yet clear whether $\hat{\mathscr{M}}$ is globally split, and if so whether a canonical splitting exists. If not, $\hat{\mathscr{M}}$ may still admit a holomorphic projection to its body which would permit one to integrate over the odd coordinates only.

There are also outstanding problems in the application of SRS techniques to superstring theory. The need for a rigorous derivation of the superstring Polyakov measure on ordinary moduli space has been reinforced by recent uncertainties about essential issues such as holomorphic factorization and would also be useful for the verification of properties like unitarity and finiteness. Further, nonperturbative questions for the superstring are even more poorly understood than for the bosonic string. Possible escapes from perturbation theory are found in the operator formalism and in generalizations of superconformal quantum field theory. It is not yet clear that SRS as presently defined are exactly the right arena for either of these. It may turn out that an elaboration of the geometrical structure is needed to accommodate the physics of picture-changing operators; such a modification might well affect the global nature of the stable-curve compactification of $\hat{\mathscr{M}}[36,37]$.

There is an emerging picture of the theory of SRS as a rich mathematical structure with a number of interesting departures from the theory of ordinary Riemann surfaces. These departures are the source of many remaining interesting questions about SRS. These include the existence of a suitable super version of

9 One can show that aut is related to the canonical bundle by aut $\cong\left(\hat{\omega}^{*}\right)^{2}[6]$ 
the Jacobian and its relation to the Picard variety, as well as issues centering on integration. Line integrals on SRS need to be better understood, and a framework for integrating the string measure on supermoduli space remains to be given [38-41].

Acknowledgements. We are grateful to R. Bott, V. Dellapietra, D. Friedan, G. Moore, M. Rothstein and C. Vafa for discussions, and to the Aspen Center for Physics for partial support. P.N. also thanks the Institut d'Etudes Scientifiques de Cargèse for hospitality while this work was being performed. This work was supported in part by NSF grant PHY82-15249, DOE contract DE-AC02-88ER40284 and by a NATO travel fellowship.

Note added in proof. Some of the exact sequences described here, as well as other points, have independently been made by Rosly, Schwarz, and Voronov [42].

\section{References}

1. Wells, R. O.: Differential analysis on complex manifolds. Berlin, Heidelberg, New York: Springer 1980

2. Alvarez-Gaume', L., Bost, J. B., Moore, G., Nelson, P., Vafa, C.: Bosonization on higher genus Riemann surfaces. Commun. Math. Phys. 112, 503 (1987)

3. Baranov, M., Shvarts, A.: Multiloop contribution in string theory. Pisma ZETF 42, 340 (1985) [= JETP Lett. 42, 419 (1986)]; Baranov, M., Manin, Yu., Frolov, I., Shvarts, A.: Multiloop contribution to fermion string. Yad. Phys. 43, 1053 (1986) [= Sov. J. Nucl. Phys. 43, 670 (1986)]

4. Friedan, D.: In Green, M., Gross, D. (eds.). Unified string theories. Singapore World Scientific 1986

5. Rabin, J., Crane, L.: Super Riemann Surfaces. Commun. Math. Phys. 113, 601 (1988)

6. Le Brun, C., Rothstein, M.: Moduli of super Riemann surfaces. Commun. Math. Phys. (in press)

7. Batchelor, M., Bryant, P.: Graded Riemann surfaces. Commun. Math. Phys. 114, 243-255 (1988)

8. Gates, S. J.: Harvard Preprint HUTP-78/A028 (1978) (unpublished); Brown, M. and Gates, S. J.: Superspace Bianchi identities and the supercovariant derivative. Ann. Phys. 122, 443 (1979)

9. Howe, P.: Super Weyl transformations in two dimensions. J. Phys. A12, 393 (1979)

10. Martinec, E.: Superspace geometry of fermionic strings. Phys. Rev. D28, 2604 (1983)

11. Brooks, R., Muhammad, F., Gates, S.: Unidexterous $D=2$ supersymmetry in superspace. Nucl. Phys. B268, 47 (1986); Moore, G., Nelson, P., Polchinski, J.: Strings and supermoduli. Phys. Lett. B169 47 (1986); Evans, M., Ovrut, B.: The world sheet supergravity of the heterotic string. Phys. Lett. 171B, 177 (1986)

12. D'Hoker, E., Phong, D.: Loop amplitudes for the fermionic string. Nucl. Phys. B278, 225 (1986)

13. Nelson, P., Moore, G.: Heterotic geometry. Nucl. Phys. B274 509 (1986)

14. Giddings, S. B., Nelson, P.: Torsion constraints and super Riemann surfaces. Phys. Rev. Lett. 59, 2619 (1987)

15. Baranov, M., Frolov, I., Schwarz, A.: Geometry of two-dimensional superconformal field theories. Teor. Mat. Fiz. 70, 92 (1987) [ = Theor. Math. Phys. 70, 64 (1987)]; Baranov, M., Schwarz, A. S.: On the multiloop contribution to string theory. Int. J. Mod. Phys. A2, 1773 (1987)

16. Nelson, P.: Holomorphic coordinates for supermoduli space. Commun. Math. Phys. 115, 167-175 (1988)

17. Leites, D.: Introduction to the theory of supermanifolds. Usp. Mat. Nauk. 35:I, 3 (1980) [ = Russ. Math. Surv. 35:I, 1 (1980)]

18. Sternberg, S.: Differential geometry, $2^{\text {d }}$ ed. New York: Chelsea 1983

19. Martin, J. L.: Proc. Roy. Soc. Lond. A251, 536 (1959); 543

20. Nelson, P.: Introduction to supermanifolds. Int. J. Mod. Phys. A3, 585 (1988)

21. Rothstein, M.: Deformations of complex supermanifolds. Proc. AMS 95, 255 (1985)

22. Gawedzki, K.: Supersymmetries. Ann. Inst. Poincaré Sect. A (NS) 27, 335 (1977)

23. Batchelor, M.: The structure of supermanifolds. Trans. Am. Math. Soc. 253, 329 (1979) 
24. Helgason, S.: Differential geometry, Lie groups, and symmetric spaces. New York: Academic 1978

25. Chern, S. S.: Complex manifolds without potential theory. $2^{\text {d }}$ ed. Berlin, Heidelberg, New York: Springer 1979

26. Manin, Yu.: Critical dimensions of string theories. Funk. Anal. 20, 88 (1986) [= Funct. Anal. Appl. 20, 244 (1987)]

27. D'Hoker, E., Phong, D.: Superholomorphic anomalies and supermoduli space. Nucl. Phys. B292, 317 (1987)

28. See, e.g., Gates, S. J., Grisaru, M., Rocek, M., Siegel, W.: Superspace. Reading, MA: Benjamin/ Cummings 1983

29. Rocek, M., van Nieuwenhuizen, P., Zhang, S.: Ann. Phys. 172, 348 (1986)

30. Spivak, M.: A comprehensive introduction to differential geometry, Vol. 1. Berkely CA: Publish or Perish 1977

31. Manin, Yu.: Gauge field theory and supersymmetry. Berlin, Heidelberg, New York: Springer 1987

32. Rothstein, M.: Integration on noncompact supermanifolds. Trans. AMS 299, 387 (1987)

33. Giddings, S. B., Nelson, P.: Line bundles on super Riemann surfaces. BUHEP-87-48 = HUTP87/A080

34. See e.g., Nelson, P.: Lectures on strings and moduli space. Phys. Rep. 149, 304 (1987)

35. Manin, Yu.: In: Arbeitstagung Bonn 1984. Lecture Notes on Mathematics, Berlin, Heidelberg, New York: Springer 1985

36. Deligne, P.: unpublished (1987)

37. Cohn, J., Friedan, D.: Modular geometry of superconformal field theory. Chicago EFI 87-07

38. Atick, J. J., Rabin, J. M., Sen, A.: An ambiguity in fermionic string perturbation theory. Nucl. Phys. B299, 279 (1988)

39. Moore, G., Morozov, A.: Some remarks on two-loop superstring calculations. IASSNS-HEP-87/47

40. Verlinde, H.: A note on the integral over the fermionic supermoduli. Utrecht preprint THU-87/26

41. Atick, J. J., Moore, G., Sen, A.: Some global issues in string perturbation theory. SLAC-PUB-4463 $=$ IASSNS-HEP-87/61

42. Rosly, A., Schwarz, A. S., Voronov, A. A.: Geometry of superconformal manifolds ITEP preprint 1987

Communicated by L. Alvarez-Gaumé

Received November 20, 1987 